\title{
HOMOGENIZATION OF DEGENERATE TWO-PHASE FLOW EQUATIONS WITH OIL TRAPPING*
}

\author{
BEN SCHWEIZER ${ }^{\dagger}$
}

\begin{abstract}
We consider the one-dimensional degenerate two-phase flow equations as a model for water drive in oil recovery. The effect of oil trapping is observed in strongly heterogeneous materials with large variations in the permeabilities and in the capillary pressure curves. In such materials, a vanishing oil saturation may appear at interior interfaces and inhibit the oil recovery. We introduce a free boundary problem that separates a critical region with locally vanishing permeabilities from a strictly parabolic region and we give a rigorous derivation of the effective conservation law.
\end{abstract}

Key words. degenerate parabolic equation, effective equations, free boundary problems, twophase flow

AMS subject classifications. 35B27, 74Q10, 35K65

DOI. $10.1137 / 060675472$

1. Introduction. The equations of two-phase flow describe the motion of two immiscible fluids in a porous medium, e.g., the flow of oil and water in rock. The equations are a challenging subject of modern analysis, in particular due to the nonlinear and degenerate coefficient functions, the permeabilities of the two phases, and the capillary pressure.

Oil trapping is an effect in media with large variations in the coefficients. Well known to experimentalists $[13,14]$, the mathematical analysis of this effect was initiated in $[5,10,9]$. Let us consider the process of oil recovery from a medium that consists of a mixture of fine and coarse materials. Starting with a high oil saturation $u$ and a high oil pressure $p$, after some time, the oil pressure falls below the entry pressure of oil in the fine material. From this point on, despite a positive saturation in the coarse material, oil can be trapped in regions that are surrounded by fine material.

In this work we analyze a one-dimensional medium that consists of two materials, distributed periodically with period $\varepsilon>0$ and with different permeabilities $k$ and different capillary pressures $p_{c}$. We denote the saturation function of the corresponding solutions by $u^{\varepsilon}$. Our aim is to find a macroscopic or effective equation, i.e., an equation that characterizes weak limits $u^{0}$ of the family $u^{\varepsilon}$ for $\varepsilon \rightarrow 0$. An effective equation allows us to determine, e.g., in a numerical scheme, the averaged profile of the solution $u^{\varepsilon}$ without resolving the scale $\varepsilon>0$.

With the method of two-scale convergence developed in [1] and measure-theoretic tools from [3] we rigorously derive the macroscopic equation

$$
\partial_{t} u^{0}+\operatorname{div} \mathcal{F}\left(u^{0}, \partial_{x} u^{0}\right)=0
$$

with a nonlinear function $\mathcal{F}$ that is determined by the coefficient functions through a finite-dimensional nonlinear problem. The effective flux function reflects the effect of oil trapping: it satisfies $\mathcal{F}(u, v)=0$ for all $v \in \mathbb{R}$ and all $u \leq u^{*} / 2$, where $u^{*}$ is the residual oil saturation in the coarse material. Our contribution continues the

\footnotetext{
*Received by the editors November 20, 2006; accepted for publication (in revised form) July 26, 2007; published electronically February 15, 2008.

http://www.siam.org/journals/sima/39-6/67547.html

${ }^{\dagger}$ Fakultät für Mathematik, TU Dortmund, Vogelpothsweg 87, D-44227 Dortmund, Germany (Ben.Schweizer@tu-dortmund.de).
} 


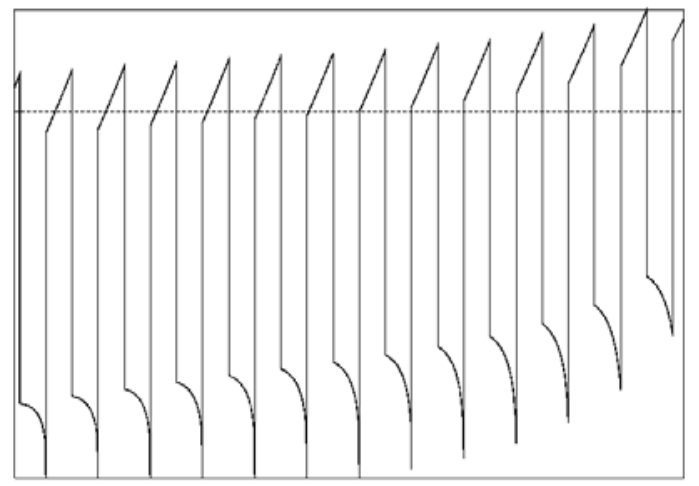

FIG. 1. Oil saturation $u^{\varepsilon}$, zoomed view. Due to different capillary pressure curves in fine and coarse material, the saturation has jumps and oil is trapped in the coarse material. The saturation vanishes at some points. At these points, the permeability degenerates, but the infinite slope $\partial_{x} u^{\varepsilon}=$ $-\infty$ makes transport of oil still possible. This figure is an unpublished numerical result and courtesy of I. S. Pop.

analysis of $[10,9]$, where effective equations were formally derived with an asymptotic expansion. In [10], which contains various scalings and treats our scaling as the "capillary limit," the authors mention the specific difficulties in the homogenization of nonlinear equations and do not attempt a rigorous derivation of the effective equations. In fact, in [9] a different law is derived by starting with another ansatz; our analysis recovers the nonlinear function of [9]. For rigorous homogenization results of nonlinear equations we refer the reader to [7] for a double porosity model, to [6] for a stochastic setting, and to $[4,17]$ for models of capillary hysteresis. All these results concern the case of nondegenerate coefficients.

We want to highlight two difficulties in the homogenization process. The first concerns the nonlinear structure of the equations: loosely speaking, fluxes are of the form $g\left(u^{\varepsilon}\right) \partial_{x} u^{\varepsilon}$. In order to pass to the two-scale limit in such a term, we need a strong convergence of the argument in the nonlinear function. The strong convergence is usually obtained from estimates for first derivatives. This procedure cannot be performed in our case, since $u^{\varepsilon}$ is an oscillatory function with jumps, and certainly not strongly convergent. The key point in the derivation of macroscopic equations in Proposition 1 is the compactness result of (3.14).

The second difficulty regards the degeneracy of the permeabilities. A strictly positive permeability $k$ results in $L^{2}$-estimates for spatial derivatives and allows us to use the compactness result. But the effect of oil trapping appears precisely in the case that, in parts of the domain, the saturation vanishes; see Figure 1. In this situation, a vanishing permeability appears and no estimate for gradients is available. Our analysis uses the technique of a free boundary description in order to proceed. We decompose the domain into a "good" region $G$ of strictly positive saturation and a "bad" region $B$; see Figure 2. We then derive the effective equations separately: in region $G$ we use two-scale convergence (Proposition 1 ) to find the effective equations in Corollary 1. In region $B$, instead, the limit equations are trivial and are derived in the form of two-sided a priori estimates. The main point is then the continuity condition across the free boundary shown in Proposition 2. This condition allows us to combine the equations again into a single equation on the whole domain. The method exploits that oscillations of the free boundary do not appear; this is ensured by the boundary and initial conditions which imply a monotonicity of the free boundary. 


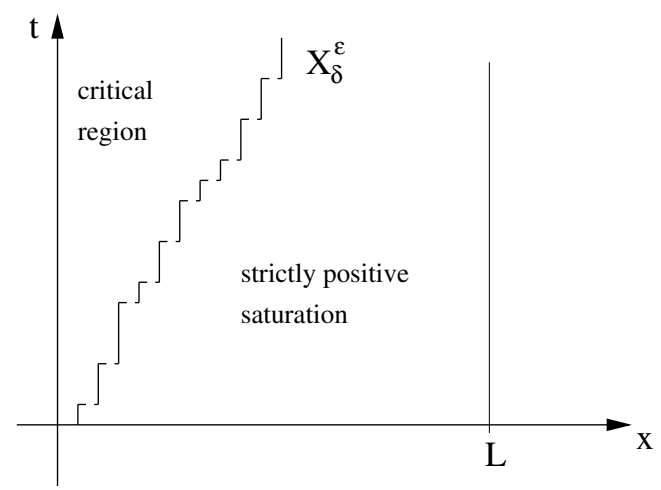

FIG. 2. A free boundary separating the critical region from a region of uniformly positive saturation and permeability. The graph illustrates that the free boundary is discontinuous for $\varepsilon>0$.

As a by-product of our description, we learn more about qualitative features of solutions. We may define an experimentally observable free boundary $X_{0}^{\varepsilon}:(0, T) \rightarrow \mathbb{R}$ as the smallest function such that in all points $(x, t)$ with $x>X_{0}^{\varepsilon}(t)$ the saturation $u^{\varepsilon}$ is strictly positive. We now ask about properties of the limiting function $\tilde{X}_{0}^{0}(t):=$ $\lim _{\varepsilon \rightarrow 0} X_{0}^{\varepsilon}(t)$. We prove in Lemma 6 that the corresponding limit curve $\{(x, t): x=$ $\left.\tilde{X}_{0}^{0}(t)\right\}$ is contained in the critical domain $B \subset \bar{\Omega}_{T}$. Thus, the effective solution provides bounds for the experimentally observable free boundary. In particular, if it can be shown that the limit equation allows only solutions $u^{0}$ with $u^{0}>u^{*} / 2$ on $\Omega_{T}$, then the experimentally observable free boundary must vanish in the limit $\varepsilon \rightarrow 0$.

Oil trapping in one-dimensional domains. We denote pressure and saturation of the oil phase by $p=p_{1}=p_{o i l}$ and $u=u_{o i l}$ and the corresponding quantities of the water phase by $p_{2}=p_{\text {water }}$ and $u_{\text {water }}=1-u$. The absolute permeability is denoted by $k$ and the relative permeabilities by $k_{r, 1}=k_{r e l, o i l}$ and $k_{r, 2}=k_{r e l, w a t e r}$. The equations in primary variables are the conservation laws for oil and water combined with the Darcy law for the velocities and the capillary pressure relation:

$$
\begin{aligned}
\partial_{t} u & =\nabla \cdot\left(k(x) k_{r, 1}(u) \nabla p_{1}\right), \\
-\partial_{t} u & =\nabla \cdot\left(k(x) k_{r, 2}(u) \nabla p_{2}\right), \\
p_{1}-p_{2} & =p_{c}(u) .
\end{aligned}
$$

Summing the conservation laws and inserting the relation between the pressure functions yields, with $K(x, u)=k(x)\left(k_{r, 1}(u)+k_{r, 2}(u)\right)$, and writing now $p$ instead of $p_{1}$,

$$
\nabla \cdot\left(K(x, u) \nabla p-k(x) k_{r, 2}(u) \nabla\left[p_{c}(u)\right]\right)=0 .
$$

One may regard this as an elliptic equation for $p$ that defines the relation between $p$ and $u$. Together with this relation, at least formally, (1.1) is an evolution equation for $u$.

In this work we study only the one-dimensional case with spatial domain $x \in \Omega=$ $(0, L)$. Equation (1.4) then implies that the expression in parentheses is constant in space. Physically, the constant describes the total flux and we write

$$
K(x, u) \partial_{x} p-k(x) k_{r, 2}(u) \partial_{x}\left[p_{c}(x, u)\right]=-q_{0} .
$$



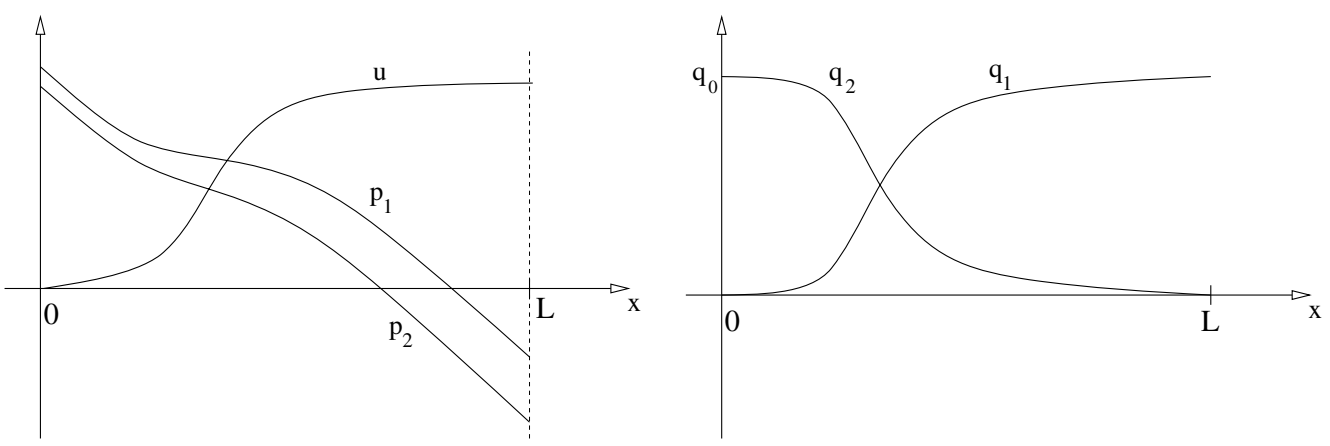

FIG. 3. Typical solutions for homogeneous materials. Left: The typical shape of oil saturation $u$, oil pressure $p_{1}$, and water pressure $p_{2}$. Right: Shape of oil flux $q_{1}=-k_{1}(x, u) \partial_{x} p_{1}$ and water flux $q_{2}=-k_{2}(x, u) \partial_{x} p_{2}$ for the chosen boundary conditions. The curves illustrate the shape for the standard equations with spatially homogeneous coefficient functions. The solutions of the effective equations in the oil trapping problem look similar, but they exhibit a residual oil saturation $u \geq u^{*} / 2$.

In order to describe imbibition from the left, we assume $q_{0}>0$. The value of $q_{0}$ is given to us by the boundary conditions. ${ }^{1}$ In order to find a single evolution equation, we solve (1.5) for $\partial_{x} p$. With the shorthand $k_{i}(x, u)=k(x) k_{r, i}(u)$ we find

$$
\partial_{x} p=-q_{0} \frac{1}{K(x, u)}+\frac{k_{2}(x, u)}{K(x, u)} \partial_{x}\left[p_{c}(x, u)\right] .
$$

Inserting into (1.1) yields

$$
\partial_{t} u=-\partial_{x}\left(f(u)-k(x) \lambda(u) \partial_{x}\left[p_{c}(x, u)\right]\right)
$$

with

$$
f(u):=q_{0} \frac{k_{r, 1}(u)}{k_{r, 1}(u)+k_{r, 2}(u)}, \quad \lambda(u):=\frac{k_{r, 1}(u) k_{r, 2}(u)}{k_{r, 1}(u)+k_{r, 2}(u)} .
$$

Equation (1.7) is an evolution equation of the form $\partial_{t} u+\partial_{x} F=0$, where $F$ is given by

$$
F(x, u):=f(u)-k(x) \lambda(u) \partial_{x}\left[p_{c}(x, u)\right] .
$$

The qualitative shape of solutions is shown in Figure 3. We emphasize that the coefficient functions are degenerate,

$$
k_{r, 1}(s) \rightarrow 0, f(s) \rightarrow 0, \lambda(s) \rightarrow 0 \text { for } s \rightarrow 0
$$

Less critical in this context is an additional degeneracy $\partial_{s} p_{c}(s) \rightarrow 0$ for $s \rightarrow 0$. Regarding high oil saturation we have $k_{r, 2}(s) \rightarrow 0$ and $\lambda(s) \rightarrow 0$ for $s \rightarrow 1$. Our interest here is in the degeneracies for $s \rightarrow 0$, and we consider a physical situation where the saturation remains bounded away from 1 for all times.

\footnotetext{
${ }^{1}$ One choice of the boundary conditions is the following. At the inlet, $x=0$, pure water enters the medium at a given rate; hence we have $u=0$ at the left boundary and $q_{0}>0$ given. At the right boundary, $x=L$, only the nonwetting fluid oil can exit; hence $k_{2}(L, u(L)) \partial_{x} p_{2}(L)=0$ or, equivalently, $-k_{1}(L, u(L)) \partial_{x} p_{1}(L)=q_{0}$. Notationally simpler is to impose a fixed saturation at the right boundary; we will therefore work with $u(0)=0$ and $p_{c}(u(L))=p_{\max }$ in what follows.
}

Copyright $@$ by SIAM. Unauthorized reproduction of this article is prohibited. 


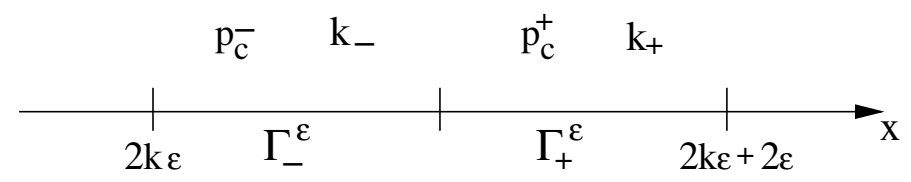

FIG. 4. Interfaces inside the material. $\Gamma_{-}^{\varepsilon}$ is a region with a fine material and $\Gamma_{+}^{\varepsilon}$ is a region with a coarse material. The permeabilities satisfy $k_{+}>k_{-}$, the capillary pressure curves $p_{c}{ }^{+}(s)<p_{c}^{-}(s)$ for all $s$.

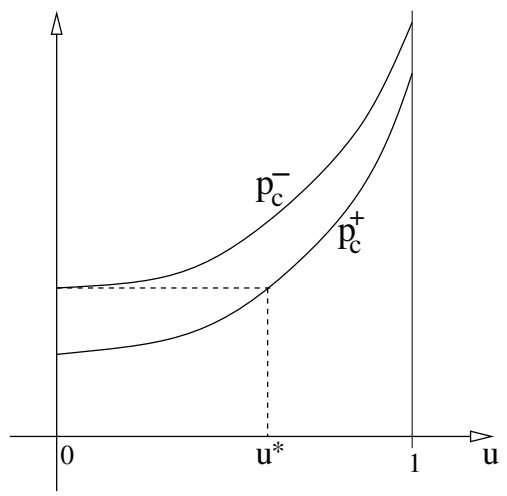

(a)

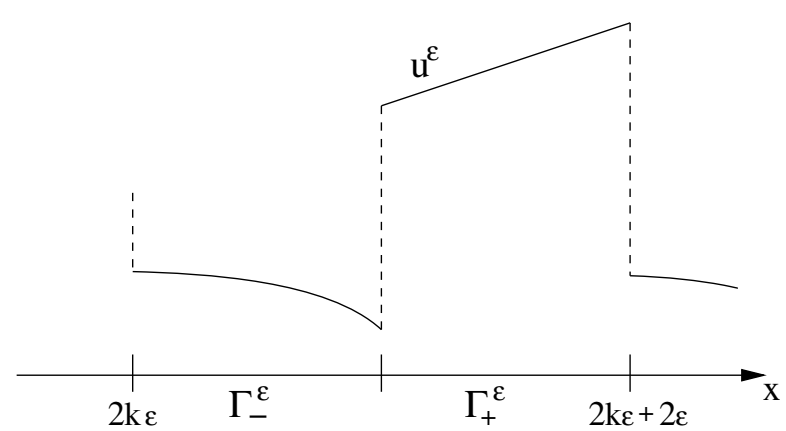

(b)

FIG. 5. (a) The graphs $p_{c}{ }^{+}$and $p_{c}{ }^{-}$. (b) Typical shape of a solution $u^{\varepsilon}(., t)$ in a small interval $(2 k \varepsilon, 2 k \varepsilon+2 \varepsilon)$. The pressures $p_{i}$ and the fluxes $q_{i}$ are almost constant, and the capillary pressure $p_{c}^{\varepsilon}\left(x, u^{\varepsilon}(x, t)\right)$ is continuous in $x$; hence the saturation $u^{\varepsilon}$ jumps from high values on $\Gamma_{+}^{\varepsilon}$ to low values on $\Gamma_{-}^{\varepsilon}$.

Oscillatory coefficient functions. In this work we are interested in oscillatory coefficients $k_{i}^{\varepsilon}=k_{i}^{0}(x / \varepsilon, u)$ and $p_{c}{ }^{\varepsilon}=p_{c}{ }^{0}(x / \varepsilon, u)$. To simplify, we consider oscillations between two different coefficient functions. We distinguish the subdomains $\Gamma_{-}^{\varepsilon}:=$ $\varepsilon(2 \mathbb{Z}+(0,1)) \cap(0, L)$ and $\Gamma_{+}^{\varepsilon}:=\varepsilon(2 \mathbb{Z}+(1,2)) \cap(0, L)$. For later use we additionally introduce $\Gamma^{\varepsilon}:=\Gamma_{+}^{\varepsilon} \cup \Gamma_{-}^{\varepsilon}$ for the spatial domain without the interfaces.

We study coefficients as sketched in Figure 4:

$$
k^{\varepsilon}(x)=\left\{\begin{array}{ll}
k_{+} & \text {for } x \in \Gamma_{+}^{\varepsilon}, \\
k_{-} & \text {for } x \in \Gamma_{-}^{\varepsilon},
\end{array} \quad p_{c}^{\varepsilon}(x, u)= \begin{cases}p_{c}^{+}(u) & \text { for } x \in \Gamma_{+}^{\varepsilon}, \\
p_{c}^{-}(u) & \text { for } x \in \Gamma_{-}^{\varepsilon} .\end{cases}\right.
$$

A typical shape of $p_{c}{ }^{ \pm}$is indicated in Figure 5(a); the corresponding local behavior of solutions is shown in Figure 5(b). The minimal pressure in $\Gamma_{-}^{\varepsilon}$ with a positive saturation is $p_{\min }^{-}=\lim _{u \backslash 0} p_{c}{ }^{-}(u)$. Of importance is the residual oil saturation $u^{*}$ in the coarse material, i.e., in $\Gamma_{+}^{\varepsilon}$. It is defined by the relation $p_{c}{ }^{+}\left(u^{*}\right)=p_{\min }^{-}$.

Our aim is to study solutions of (1.7) for this choice of coefficients. Understanding the equations in the distributional sense means to demand at the interfaces $\xi \in \varepsilon \mathbb{Z}$ the continuity of flux and capillary pressure. Since the capillary pressure curves are multivalued in general, we demand for all $\xi \in \varepsilon \mathbb{Z}$

$$
\begin{aligned}
& F(\xi-0, u(\xi-0))=F(\xi+0, u(\xi+0)), \\
& p_{c}(\xi-0, u(\xi-0)) \cap p_{c}(\xi+0, u(\xi+0)) \neq \emptyset .
\end{aligned}
$$

Here, we use the notation $h(x \pm 0)$ for $\lim _{ \pm \delta \backslash 0} h(x+\delta)$, or, if $h \in H^{1}$, for the corresponding trace. Relation (1.10) is a compact way to write the standard interface 
condition for the capillary pressure which was derived in [5]. We use set-valued capillary pressure functions that assign to the saturations $s=0$ and $s=1$ an interval, e.g., $p_{c}{ }^{-}(\xi, 0):=\left(-\infty, p_{\text {min }}^{-}\right]$for $\xi \in \Gamma_{-}^{\varepsilon}$. In this way, if the saturation vanishes at one side of the interface, the pressure at the other side must be below the entry pressure $p_{\min }$, but the exact value is not determined. The classical description of (1.10) is that we necessarily are in one of the following situations: (a) at both sides, the saturation is strictly between 0 and 1 , and the capillary pressures on both sides coincide; (b) we have $s=0$ at side $A, s \in(0,1)$ at side $B$, and there holds $p_{c} \leq p_{\min }$, where $p_{c}$ is evaluated at side $B$ and $p_{\min }$ at side $A$; (c) we have $s=0$ at both sides; (d) an analogous case with $s=1$ on one side.

In the next step we write the equations in a compact and symmetric form. The conservation law (1.7) is recovered in (1.11) with $g^{ \pm}(u):=k_{ \pm} \lambda(u) \partial_{u} p_{c}{ }^{ \pm}(u)$ and $f^{ \pm}(u):=f(u)$.

Mathematical description and main result. We assume that the coefficients are $x$-independent on each set $\Gamma_{ \pm}^{\varepsilon}$,

$$
g^{\varepsilon}(x, u):=\left\{\begin{array}{ll}
g^{+}(u) & \text { for } x \in \Gamma_{+}^{\varepsilon}, \\
g^{-}(u) & \text { for } x \in \Gamma_{-}^{\varepsilon},
\end{array} \quad f^{\varepsilon}(x, u):= \begin{cases}f^{+}(u) & \text { for } x \in \Gamma_{+}^{\varepsilon}, \\
f^{-}(u) & \text { for } x \in \Gamma_{-}^{\varepsilon}\end{cases}\right.
$$

We study the conservation law

$$
\begin{aligned}
& \partial_{t} u^{\varepsilon}+\partial_{x} F^{\varepsilon}=0 \text { on } \Gamma^{\varepsilon}, \\
& F^{\varepsilon}=f^{\varepsilon}\left(x, u^{\varepsilon}\right)-g^{\varepsilon}\left(x, u^{\varepsilon}\right) \partial_{x} u^{\varepsilon}, \\
& F^{\varepsilon} \text { and } p_{c}{ }^{\varepsilon}\left(x, u^{\varepsilon}\right) \text { are continuous in } \mathbb{Z} \varepsilon .
\end{aligned}
$$

Here, the continuity is understood in the classical sense for $F^{\varepsilon}$, and in the sense of (1.10) for $p_{c}{ }^{\varepsilon}$. From now on, we study solution sequences $u^{\varepsilon}$ to this equation, complemented with the initial condition $\left.p_{c}^{\varepsilon}\left(u^{\varepsilon}\right)\right|_{t=0}=p_{\max }$ on $(0, L)$ for some initial pressure value $p_{\max } \in\left(p_{c}{ }^{-}(0), p_{c}{ }^{+}(1)\right)$. As boundary conditions we impose $u^{\varepsilon}(0, t)=$ 0 and $p_{c}{ }^{\varepsilon}\left(u^{\varepsilon}(L, t)\right)=p_{\max }$ for all $t \in(0, T)$. Throughout we assume the following monotonicity and regularity of the coefficient functions.

Assumptions. On flux and diffusivity we assume $0 \leq f^{ \pm} \in C^{0}([0,1], \mathbb{R}), 0 \leq$ $g^{ \pm} \in C^{0,1}([0,1], \mathbb{R}), f^{-}(0)=g^{-}(0)=0$. Furthermore, $f^{ \pm} \leq c g^{ \pm}$on the interval $\left[0,\left(p_{c}{ }^{+}\right)^{-1}\left(p_{\max }\right)\right]$ for some constant $c>0$, and $f^{ \pm}>0$ on $(0,1)$. On the capillary pressure we assume $p_{c}{ }^{+} \leq p_{c}{ }^{-}$with strictly monotone functions $p_{c}{ }^{ \pm} \in C^{1}([0,1], \mathbb{R})$.

Our main theorem is the rigorous derivation of effective equations. They are characterized by a nonlinear flux function $\mathcal{F}:[0,1] \times \mathbb{R} \rightarrow \mathbb{R}$, which is constructed in (3.1)-(3.7). For the particular choice of coefficients considered there, our flux function $\mathcal{F}$ coincides with that of $[9]$.

THEOREM 1. Let $\left(u^{\varepsilon}, F^{\varepsilon}\right)$ be a family of entropy solutions to (1.11) on $\Omega_{T}=$ $(0, L) \times(0, T)$, as in Definition 1 of section 2 , satisfying the above boundary conditions. Then, for a subsequence $\varepsilon \rightarrow 0$ and for appropriate limiting functions, we find

$$
u^{\varepsilon} \rightarrow u^{0} \text { in } L^{\infty}\left(\Omega_{T}\right) \text { weak-ᄎ, } \quad F^{\varepsilon} \rightarrow F^{0} \text { in } L^{2}\left(\Omega_{T}\right) \text { weakly. }
$$

The limits satisfy the conservation law

$$
\partial_{t} u^{0}+\partial_{x} F^{0}=0
$$


in the distributional sense on $\Omega_{T}$. The limit $u^{0} \in L^{\infty}\left(\Omega_{T}\right)$ has the distributional derivative $\partial_{x} u^{0} \in L^{1}\left(\Omega_{T}\right)$. The flux satisfies the relation

$$
F^{0}=\mathcal{F}\left(u^{0}, \partial_{x} u^{0}\right) \text { almost everywhere in } \Omega_{T}
$$

with the nonlinear function $\mathcal{F}\left(u, \partial_{x} u\right)=\mathcal{F}_{0}(u)-\mathcal{D}(u) \partial_{x} u$ defined in $(3.1)-(3.7)$.

The remaining sections are devoted to the proof of Theorem 1 and are organized as follows. In section 2 we construct entropy solutions and derive comparison and monotonicity results. Section 3 is devoted to a two-scale homogenization result for regions with a strictly positive saturation. The homogenization in the general situation with degenerate solutions is performed in section 4 . We introduce a description with a free boundary, derive the effective equations in the critical region, and determine the continuity condition across the free boundary.

The analysis of the limit problem (1.12), (1.13) is not the aim of this contribution. Interesting questions concern the existence and uniqueness of solutions to this degenerate problem, and the position of the free boundary $X(t):=\sup \left\{x: u^{0}(x, t)=u^{*} / 2\right\}$. We note that the results of [2] cannot be applied to the equations in the above form, since, for a degenerate function $\mathcal{D}(u)$, the ellipticity assumption (Assumption 1.1(3)) of [2] is not satisfied. But the special structure $\mathcal{F}\left(u, \partial_{x} u\right)=\mathcal{F}_{0}(u)-\mathcal{D}(u) \partial_{x} u$ allows us to introduce formally a new variable $U$ with $\partial_{x} U=\mathcal{D}(u) \partial_{x} u$ such that with $u=b(U)$ and $a\left(u, \partial_{x} U\right)=-\mathcal{F}_{0}(u)+\partial_{x} U$, the results of [2] and [15] may be applicable. The appearance of free boundaries is well known in porous-media-type equations; we refer the reader to [11] for results on the one-dimensional degenerate Cauchy problem $\partial_{t} u=\partial_{x}^{2}[a(u)]+\partial_{x}[b(u)]$ regarding existence, uniqueness, regularity, and speed of propagation of the free boundary.

Notation. The value of the constant $C$ in estimates may change from one line to the next. For a set $Q$ the function $\mathbf{1}_{Q}$ denotes the characteristic function $\mathbf{1}_{Q}(x)=1$ for $x \in Q$ and $\mathbf{1}_{Q}(x)=0$ for $x \notin Q$.

\section{Entropy solutions and monotonicity.}

2.1. Entropy solutions and regularity. In this section we sketch a solution concept that allows us to derive comparison principles for solutions. For other existence and uniqueness results we refer the reader to [8] and [12], where methods of [2] are extended to two-phase flow. An existence proof that uses a smoothing of the jump condition is performed in [5]. We refer the reader to [16] for a discussion of approximation schemes to degenerate equations that are also used below.

We always assume $p_{\max } \in\left(p_{c}{ }^{-}(0), p_{c}{ }^{+}(1)\right)$ and consider only boundary conditions as described above. We use the notion of a family of regularized equations: We assume that, for a sequence $\eta \searrow 0$, we have coefficient functions $g_{\eta}^{ \pm} \in C^{1}([0,1], \mathbb{R}), f_{\eta}^{ \pm}=f^{ \pm}$, and $p_{c}{ }^{ \pm, \eta} \in C^{1}([0,1], \mathbb{R})$ that are strictly monotone and satisfy the same inequalities as the original coefficients. The equations are regularized in the sense that $g_{\eta}^{ \pm} \geq \eta, p_{c}{ }^{ \pm, \eta}$ is single valued, and $p_{c}{ }^{-, \eta}(0)=p_{c}{ }^{+, \eta}(0)$. They approximate the original equation in the sense that $p_{c}{ }^{+, \eta}=p_{c}{ }^{+}$on $(0,1), g_{\eta}^{ \pm} \rightarrow g^{ \pm}$uniformly on $[0,1]$, and $p_{c}{ }^{-, \eta} \rightarrow p_{c}{ }^{-}$ uniformly on compact subsets of $(0,1]$. By an appropriate choice of the regularization we can additionally achieve that the family $g_{\eta}^{ \pm} \partial_{u} p_{c}{ }^{ \pm, \eta}$ is uniformly bounded on $[0,1]$.

In the following definition we use the primitive $G^{\varepsilon}(x, u)$ of $g^{\varepsilon}$, i.e., the function with $\partial_{u} G^{\varepsilon}(x, u)=g^{\varepsilon}(x, u)$ and $G^{\varepsilon}(x, 0)=0$. Since $g^{\varepsilon}(., u)$ is piecewise constant, we can interpret the term $g^{\varepsilon}\left(u^{\varepsilon}\right) \partial_{x} u^{\varepsilon}$ as the distribution $\left.\partial_{x} G^{\varepsilon}\left(x, u^{\varepsilon}\right)\right|_{\Gamma^{\varepsilon} \times(0, T)}$. Since we will work with $\partial_{x} G^{\varepsilon}(x, u) \mathbf{1}_{\Gamma^{\varepsilon}}(x) \in L^{2}\left(\Omega_{T}\right)$, we have well-defined traces $\left.G^{\varepsilon}\left(x, u^{\varepsilon}\right)\right|_{\partial \Gamma^{\varepsilon} \times(0, T)}$. Since $G^{\varepsilon}(x,$.$) is invertible, this defines also traces of u^{\varepsilon}$ and gives a precise meaning to the interface conditions.

Copyright $@$ by SIAM. Unauthorized reproduction of this article is prohibited. 
We write the interface condition (1.10) in a more reader-friendly form, considering $p_{c}{ }^{-}$as multivalued and $p_{c}{ }^{+}$as a function.

Definition 1. A saturation-flux pair $\left(u^{\varepsilon}, F^{\varepsilon}\right)$ is a weak solution of (1.11) on $\Omega_{T}=(0, L) \times(0, T)$ if $u^{\varepsilon} \in L^{\infty}\left(\Omega_{T},[0,1]\right)$ and $F^{\varepsilon} \in L^{2}\left(\Omega_{T}\right)$ satisfy

$$
\begin{aligned}
\partial_{t} u^{\varepsilon}+\partial_{x} F^{\varepsilon} & =0 & & \text { in } \mathcal{D}^{\prime}\left(\Omega_{T}\right), \\
F^{\varepsilon} & =f^{\varepsilon}\left(u^{\varepsilon}\right)-\partial_{x}\left[G^{\varepsilon}\left(u^{\varepsilon}\right)\right] & & \text { in } \mathcal{D}^{\prime}\left(\Gamma^{\varepsilon} \times(0, T)\right), \\
p_{c}{ }^{+}\left(u^{\varepsilon}(2 k \varepsilon-0)\right) & \in p_{c}{ }^{-}\left(u^{\varepsilon}(2 k \varepsilon+0)\right) & & \forall k \in \mathbb{Z}, 2 k \varepsilon \in(0, L), \\
p_{c}{ }^{+}\left(u^{\varepsilon}(2 k \varepsilon+\varepsilon+0)\right) & \in p_{c}{ }^{-}\left(u^{\varepsilon}(2 k \varepsilon+\varepsilon-0)\right) & & \forall k \in \mathbb{Z}, 2 k \varepsilon \in(0, L) .
\end{aligned}
$$

A weak solution $\left(u^{\varepsilon}, F^{\varepsilon}\right)$ is called an entropy solution if there exists a family of regularized equations and a corresponding family of solutions $\left(u_{\eta}^{\varepsilon}, F_{\eta}^{\varepsilon}\right)$ with $u_{\eta}^{\varepsilon} \rightarrow u^{\varepsilon}$ in $L^{2}\left(\Omega_{T}\right)$ and $F_{\eta}^{\varepsilon} \rightarrow F^{\varepsilon}$ in $L^{2}\left(\Omega_{T}\right)$ for $\eta \rightarrow 0$.

Lemma 1 (existence and a priori estimate). For every $\varepsilon>0$, there exists an entropy solution $\left(u^{\varepsilon}, F^{\varepsilon}\right)$. With a constant $C$ independent of $\varepsilon$ there hold $\left\|F^{\varepsilon}\right\|_{L^{2}\left(\Omega_{T}\right)} \leq$ $C$ and the following regularity on the domain of positive saturation: For all $\delta>0$ there exists $C_{\delta}$ independent of $\varepsilon$ such that

$$
\int_{0}^{T} \int_{0}^{L}\left|\partial_{x} u^{\varepsilon}\right|^{2} \mathbf{1}_{\left\{u^{\varepsilon} \geq \delta\right\}} \mathbf{1}_{\Gamma^{\varepsilon}} \leq C_{\delta}
$$

Proof. The regularized system $(\eta>0)$ is a parabolic problem with finitely many transmission points and can be solved by standard methods. The maximum principle implies the bounds $0 \leq u_{\eta}^{\varepsilon} \leq 1$. They allow us to select a subsequence $\eta \rightarrow 0$ and a weak $L^{2}\left(\Omega_{T}\right)$-limit $u^{\varepsilon}$. The monotonicity in $t$, shown in Lemma 3 , implies the boundedness of $\partial_{t} u_{\eta}^{\varepsilon} \in L^{1}\left(\Omega_{T}\right)$. Estimate $(2.1)$ for $\eta>0$ provides uniform bounds for the positive part $\left(u_{\eta}^{\varepsilon}-\delta\right)_{+} \in L^{2}\left((0, T), H^{1}\left(\Gamma^{\varepsilon}\right)\right)$; hence the sequence $\left(u_{\eta}^{\varepsilon}-\delta\right)_{+}$ is precompact in $L^{2}\left(\Omega_{T}\right)$ for every $\delta>0$. Choosing a diagonal sequence we find a subsequence with $u_{\eta}^{\varepsilon} \rightarrow u^{\varepsilon}$ strongly in $L^{2}\left(\Omega_{T}\right)$.

Below we derive an estimate for the sequence $F_{\eta}^{\varepsilon} \in L^{2}\left(\Omega_{T}\right)$ and thus, by boundedness of $f^{\varepsilon}$, there is also a uniform bound for $\partial_{x} G_{\eta}^{\varepsilon}\left(x, u_{\eta}^{\varepsilon}\right) \mathbf{1}_{\Gamma^{\varepsilon}} \in L^{2}\left(\Omega_{T}\right)$. We can choose a weakly convergent subsequence $F_{\eta}^{\varepsilon} \rightarrow F^{\varepsilon}$ in $L^{2}\left(\Omega_{T}\right)$ and find also $G_{\eta}^{\varepsilon}\left(u_{\eta}^{\varepsilon}\right) \rightarrow G^{\varepsilon}\left(u^{\varepsilon}\right)$ by the strong convergence of $u_{\eta}^{\varepsilon}$. This implies that the pair $\left(u^{\varepsilon}, F^{\varepsilon}\right)$ solves the conservation law and the characterization of $F^{\varepsilon}$ of the second equation.

The solutions of the regularized problems satisfy the interface inclusions as equalities and we have a weak convergence of $\partial_{x} G\left(u_{\eta}^{\varepsilon}\right)$. The trace theorem implies that the limit $u^{\varepsilon}$ satisfies again the interface conditions, and hence it is a weak solution of (1.11).

We now verify the a priori estimates, omitting everywhere the index $\eta>0$. We multiply the conservation law

$$
\partial_{t} u^{\varepsilon}+\partial_{x}\left[f\left(u^{\varepsilon}\right)-g^{\varepsilon}\left(u^{\varepsilon}\right) \partial_{x} u^{\varepsilon}\right]=0 \text { on } \Gamma^{\varepsilon}
$$

by the continuous function $p_{c}{ }^{\varepsilon}\left(u^{\varepsilon}\right)-p_{\max }$ and integrate by parts. Interior boundary integrals vanish due to the continuity of the flux. The right boundary integral vanishes because of the boundary condition $p_{c}^{\varepsilon}\left(L, u^{\varepsilon}(L, t)\right)=p_{\max }$. We obtain

$$
\begin{aligned}
\int_{0}^{T} & \int_{\Gamma^{\varepsilon}}\left[p_{c}^{\varepsilon}\left(u^{\varepsilon}\right)-p_{\text {max }}\right] \partial_{t} u^{\varepsilon}+\int_{0}^{T} \int_{\Gamma^{\varepsilon}} g^{\varepsilon}\left(u^{\varepsilon}\right) \partial_{x} u^{\varepsilon} \partial_{x}\left[p_{c}^{\varepsilon}\left(u^{\varepsilon}\right)\right] \\
= & \int_{0}^{T} \int_{\Gamma^{\varepsilon}} f^{\varepsilon}\left(u^{\varepsilon}\right) \partial_{x}\left[p_{c}^{\varepsilon}\left(u^{\varepsilon}\right)\right]+\int_{0}^{T}\left[f\left(u^{\varepsilon}\right)-g^{\varepsilon}\left(u^{\varepsilon}\right) \partial_{x} u^{\varepsilon}\right]_{x=0}\left(p_{c}^{-, \eta}(0)-p_{\max }\right) .
\end{aligned}
$$

Copyright (c) by SIAM. Unauthorized reproduction of this article is prohibited. 
For the flux at the left boundary, which appears in the last integral, we calculate, using $\varphi(x, t)=L-x$,

$$
\begin{aligned}
& -L \int_{0}^{T}\left[f\left(u^{\varepsilon}\right)-g^{\varepsilon}\left(u^{\varepsilon}\right) \partial_{x} u^{\varepsilon}\right]_{x=0}=\int_{0}^{T} \int_{0}^{L} \partial_{x}\left(\left[f\left(u^{\varepsilon}\right)-g^{\varepsilon}\left(u^{\varepsilon}\right) \partial_{x} u^{\varepsilon}\right] \varphi\right) \\
& \quad=\int_{0}^{T} \int_{0}^{L} \partial_{x}\left[f\left(u^{\varepsilon}\right)-g^{\varepsilon}\left(u^{\varepsilon}\right) \partial_{x} u^{\varepsilon}\right] \varphi-\int_{0}^{T} \int_{0}^{L}\left[f\left(u^{\varepsilon}\right)-g^{\varepsilon}\left(u^{\varepsilon}\right) \partial_{x} u^{\varepsilon}\right] \\
& \quad=-\int_{0}^{T} \int_{0}^{L} \partial_{t} u^{\varepsilon} \varphi-\int_{0}^{T} \int_{0}^{L}\left[f\left(u^{\varepsilon}\right)-g^{\varepsilon}\left(u^{\varepsilon}\right) \partial_{x} u^{\varepsilon}\right] \\
& \quad \leq L^{2}+C+\int_{\Omega_{T}} g^{\varepsilon}\left(u^{\varepsilon}\right)\left|\partial_{x} u^{\varepsilon}\right| .
\end{aligned}
$$

We continue the above calculation, exploiting that $p_{c}{ }^{\varepsilon}\left(u^{\varepsilon}\right) \partial_{t} u^{\varepsilon}$ is the time derivative of a bounded function, and use the uniform positivity $\partial_{u} p_{c}{ }^{\varepsilon} \geq c_{0}>0$ and the bound $f^{ \pm} \leq c g^{ \pm}:$

$$
\int_{0}^{T} \int_{\Gamma^{\varepsilon}} g^{\varepsilon}\left(u^{\varepsilon}\right) \partial_{u} p_{c}^{\varepsilon}\left(u^{\varepsilon}\right)\left|\partial_{x} u^{\varepsilon}\right|^{2} \leq C+C \int_{0}^{T} \int_{\Gamma^{\varepsilon}} g^{\varepsilon}\left(u^{\varepsilon}\right) \partial_{u} p_{c}{ }^{\varepsilon}\left(u^{\varepsilon}\right)\left|\partial_{x} u^{\varepsilon}\right| .
$$

Application of the Cauchy-Schwarz inequality yields, by the boundedness of $g^{\varepsilon} \partial_{u} p_{c}{ }^{\varepsilon}$, a bound for the left-hand side, independent of $\varepsilon$ and $\eta$. Exploiting once more $\partial_{u} p_{c}{ }^{\varepsilon} \geq$ $c_{0}$ and $g^{ \pm}>0$ on $(0,1]$, this implies (2.1). It furthermore shows that the family $F^{\varepsilon}=\left[f\left(u^{\varepsilon}\right)-g^{\varepsilon}\left(x, u^{\varepsilon}\right) \partial_{x} u^{\varepsilon}\right] \mathbf{1}_{\Gamma^{\varepsilon}}$ is uniformly bounded in $L^{2}\left(\Omega_{T}\right)$.

2.2. Comparison principles and monotonicity. In this subsection we derive results for entropy solutions of (1.11) and assume always the above boundary conditions and the initial condition $p_{c}^{\varepsilon}\left(u^{\varepsilon}(., 0)\right)=p_{\max }$ on $(0, L)$ for $p_{\max } \in$ $\left(p_{c}{ }^{-}(0), p_{c}{ }^{+}(1)\right)$.

LEmma 2 (lower bound for $u^{\varepsilon}$ ). There exist $\delta_{0}>0$ and $\varepsilon_{0}>0$ such that for all $\delta \in\left(0, \delta_{0}\right)$ and $\varepsilon \in\left(0, \varepsilon_{0}\right)$ the following holds. Let $(a, b) \subset(0, L)$ with $a, b \in 2 \varepsilon \mathbb{Z}+\varepsilon$, and let $u^{\varepsilon}$ be an entropy solution of (1.11) with

$$
u^{\varepsilon}(a-0, t) \geq \delta, u^{\varepsilon}(b-0, t) \geq \delta \quad \forall t \in\left(0, t_{0}\right) .
$$

Then there holds $u^{\varepsilon} \geq \delta$ on $(a, b) \times\left(0, t_{0}\right)$.

Proof. It is sufficient to show the claim for the regularized solutions $u_{\eta}^{\varepsilon}$ and to take the limit $\eta \rightarrow 0$. We therefore perform all calculations for the more regular solutions $u_{\eta}^{\varepsilon}$, but we omit in what follows the fixed index $\eta$.

Our aim is to construct a $2 \varepsilon$-periodic stationary subsolution $U(y)=U_{\delta}^{\varepsilon}(y) \geq \delta$ for $y \in[a, b]$. For a parameter $q \in \mathbb{R}_{+}$which denotes the constant flux of the subsolution, we define $U=U(., q):[0,2 \varepsilon] \rightarrow \mathbb{R}, y \mapsto U(y)$ as the solution of

$$
\begin{aligned}
& f^{\varepsilon}(y, U(y))-g^{\varepsilon}(y, U(y)) \partial_{y} U(y)=q \text { in }(0, \varepsilon) \cup(\varepsilon, 2 \varepsilon), \\
& U(\varepsilon-0)=\delta, \quad U(\varepsilon+0)=\left(p_{c}^{+}\right)^{-1}\left(p_{c}^{-}(\delta)\right) .
\end{aligned}
$$

We note that, by the positivity of $g^{\varepsilon},(2.2)$ is an ordinary differential equation which can be solved locally with the boundary condition (2.3). The smallness of $\varepsilon>0$ and the fact that $q-f^{\varepsilon}(y, \delta)$ becomes positive for $\delta \rightarrow 0$ imply that solutions can be defined on the whole intervals $(0, \varepsilon)$ and $(\varepsilon, 2 \varepsilon)$. The solution operator defines a family of functions $U(., q)$ and the continuous function $G=G_{\delta}: \mathbb{R}_{+} \rightarrow \mathbb{R}$ :

$$
G(q):=p_{c}{ }^{+}(U(2 \varepsilon-0))-{p_{c}}^{-}(U(0+0))
$$

Copyright (c) by SIAM. Unauthorized reproduction of this article is prohibited. 
Our aim is to choose the parameter $q=q^{*}$ with $G\left(q^{*}\right)=0$ such that the function $U=U\left(., q^{*}\right)$ can be extended to a $2 \varepsilon$-periodic stationary solution of (1.11).

For two special values of $q$ we can evaluate the sign of $G(q)$. For $q_{1}=f^{-}(\delta)$, the solution $U\left(., q_{1}\right)=\delta$ is constant on the interval $(0, \varepsilon)$. On the interval $(\varepsilon, 2 \varepsilon)$ the derivative $\partial_{y} U$ is positive by the positivity of $f^{+}\left(u^{*}\right)$; here we exploit the continuity of $f^{ \pm}, f^{-}(0)=0$ and the smallness of $\delta$ and $\varepsilon$. We find that

$$
G\left(q_{1}\right) \geq p_{c}{ }^{+}(U(\varepsilon+0))-p_{c}{ }^{-}(\delta)=0 .
$$

On the other hand, for $q_{2}=f^{+}(U(\varepsilon+0))$, the solution $U\left(., q_{2}\right)$ is constant on the interval $(\varepsilon, 2 \varepsilon)$ and decreasing on $(0, \varepsilon)$. Monotonicity of $p_{c}{ }^{ \pm}$implies $G\left(q_{2}\right) \leq 0$. By continuity, there is a critical value $q^{*}=q^{*}(\delta, \varepsilon) \in\left[q_{1}, q_{2}\right]$ with $G\left(q^{*}\right)=0$, and we use $U=U\left(., q^{*}\right)$ in what follows.

We now define the subsolution $U^{\varepsilon}(x, t)$ as the $2 \varepsilon$-periodic continuation of $U_{\delta e^{-\lambda t}}^{\varepsilon}(x)$, where we replaced $\delta$ by $\delta e^{-\lambda t}$ for a small constant $\lambda>0$. We claim that $u^{\varepsilon}$ can never touch the subsolution $U^{\varepsilon}$. Because $\partial_{t} U^{\varepsilon}<0$, this is the standard comparison principle for all points $x$ that are not contained in $\varepsilon \mathbb{Z}$. Let us assume that $t>0$ is the first time instance at which the solutions touch each other and that, for a point $x \in \varepsilon \mathbb{Z}$, we have $u^{\varepsilon}(x+0, t)=U^{\varepsilon}(x+0, t)$. Since $u^{\varepsilon}$ and $U^{\varepsilon}$ both satisfy the $p_{c}$-jump condition, there also holds $u^{\varepsilon}(x-0, t)=U^{\varepsilon}(x-0, t)$. Then, because $u^{\varepsilon}(., t) \geq U^{\varepsilon}(., t)$, we have $\partial_{x} u^{\varepsilon}(x+0, t) \geq \partial_{x} U^{\varepsilon}(x+0, t)$ and $\partial_{x} u^{\varepsilon}(x-0, t) \leq \partial_{x} U^{\varepsilon}(x-0, t)$. The subsolution $U^{\varepsilon}$ has the continuous flux $q^{*}\left(\delta e^{-\lambda t}, \varepsilon\right)$ and $u^{\varepsilon}$ also has a continuous flux; since also $f\left(u^{\varepsilon}\right)=f\left(U^{\varepsilon}\right)$ and the same for $g$ in the point $x$, the derivatives must coincide, $\partial_{x} u^{\varepsilon}(x+0, t)=\partial_{x} U^{\varepsilon}(x+0, t)$. As a consequence, also the fluxes of $u^{\varepsilon}$ and $U^{\varepsilon}$ coincide in $x$. In $(x+0, t)$ it holds that

$$
-\partial_{x}\left[f^{\varepsilon}\left(u^{\varepsilon}\right)-g^{\varepsilon}\left(u^{\varepsilon}\right) \cdot \partial_{x} u^{\varepsilon}\right]=\partial_{t} u^{\varepsilon}<0=-\partial_{x}\left[f^{\varepsilon}\left(U^{\varepsilon}\right)-g^{\varepsilon}\left(U^{\varepsilon}\right) \cdot \partial_{x} U^{\varepsilon}\right],
$$

and hence $\partial_{x}^{2} u^{\varepsilon}<\partial_{x}^{2} U^{\varepsilon}$, which is a contradiction to $u^{\varepsilon} \geq U^{\varepsilon}$.

LEMMA 3 (monotonicity of $u^{\varepsilon}$ ). Let $u^{\varepsilon}$ be an entropy solution of (1.11) as above. Then the following hold:

1. Decay in time. The map $t \mapsto u^{\varepsilon}(x, t)$ is monotonically nonincreasing for almost every $x$.

2. Monotonicity in space. The map $k \mapsto u^{\varepsilon}(2 k \varepsilon+y, t)$ is monotonically nondecreasing for every $t \in(0, T)$ and every $y \in[0,2 \varepsilon]$.

Proof. As in the last proof, it suffices to verify the monotonicity for the approximate solutions $u=u_{\eta}^{\varepsilon}$. We therefore study, for the strictly positive coefficient $g=g(x, u) \geq \eta$ and the strictly monotone single-valued function $p_{c}=p_{c}(x, u)$, solutions $u$ of

$$
\begin{aligned}
\partial_{t} u+\partial_{x}\left(f(x, u)-g(x, u) \partial_{x} u\right) & =0, \\
{\left[p_{c}(x, u)\right] } & =0, \\
{\left[f(x, u)-g(x, u) \partial_{x} u(x)\right] } & =0,
\end{aligned}
$$

where the last two relations hold in points $x \in \mathbb{Z} \varepsilon$. By regularity theory for strictly parabolic equations we may assume that $u$ is a classical solution of the above system.

Proof of part 1 . We claim that $\partial_{t} u \leq 0$ holds on $G$. Indeed, the function $v=-\partial_{t} u$ is nonnegative at $t=0$ and the equations are, with $\kappa=0$,

$$
\begin{aligned}
\partial_{t} v+\partial_{x}\left(f_{u}(x, u) \cdot v-g_{u}(x, u) \cdot v \partial_{x} u-g(x, u) \partial_{x} v\right) & =\kappa, \\
{\left[\partial_{u} p_{c}(x, u) \cdot v\right] } & =0, \\
{\left[f_{u}(x, u) \cdot v-g_{u}(x, u) \cdot v \partial_{x} u(x)-g(x, u) \partial_{x} v(x)\right] } & =0 .
\end{aligned}
$$

Copyright (c) by SIAM. Unauthorized reproduction of this article is prohibited. 
The boundary conditions are $v(0, t)=v(L, t)=0$ for all $t \in(0, T)$. In order to show that $v$ remains nonnegative, it suffices to show that the solutions $v_{\kappa}$ of the above system with small right-hand side $\kappa \in \mathbb{R}, \kappa>0$, remain nonnegative.

Let $t>0$ be the first time instance such that $v_{\kappa}(., t)$ has a zero in $x$. By the standard comparison principle, the zero cannot be in $(0, L) \backslash \varepsilon \mathbb{Z}$. Let us therefore assume $x \in \varepsilon \mathbb{Z}$. The first jump condition implies that, with $v_{\kappa}$ vanishing on one side of $x$, it vanishes on both sides. Continuity implies $v_{\kappa}(., t) \geq 0$, and hence we have the geometric conditions $\partial_{x} v_{\kappa}(x-0, t) \leq 0$ and $\partial_{x} v_{\kappa}(x+0, t) \geq 0$. The second jump condition then implies that $\partial_{x} v_{\kappa}=0$ from both sides. The geometric condition $\partial_{t} v_{\kappa}(x, t) \leq 0$, together with $\kappa>0$, implies

$$
\partial_{x}\left(f_{u}(x, u) \cdot v_{\kappa}-g_{u}(x, u) \cdot v_{\kappa} \partial_{x} u-g(x, u) \partial_{x} v_{\kappa}\right)>0
$$

in the vicinity of $x$. We conclude that $\partial_{x}^{2} v_{\kappa}<0$ and thus a contradiction to $v_{\kappa} \geq 0$.

Proof of part 2. We claim that the function $v(x, t)=u(x+2 \varepsilon, t)-u(x, t)$, defined on $(0, L-2 \varepsilon) \times[0, T]$, is nonnegative for all times. Indeed, $v(., 0)=0$ initially, and there hold $v(0, t) \geq 0$ and $v(L-2 \varepsilon, t) \geq 0$ for all $t \in(0, T)$. With $u_{+}(x, t):=u(x+2 \varepsilon, t)$, the equations for $v=u_{+}-u$ read

$$
\begin{aligned}
\partial_{t} v+\partial_{x}\left(f\left(x, u_{+}\right)-f(x, u)-g\left(x, u_{+}\right) \partial_{x} u_{+}+g(x, u) \partial_{x} u\right) & =0, \\
{\left[p_{c}\left(x, u_{+}\right)-p_{c}(u)\right] } & =0, \\
{\left[f\left(x, u_{+}\right)-g\left(x, u_{+}\right) \partial_{x} u_{+}(x)-f(x, u)+g(x, u) \partial_{x} u(x)\right] } & =0 ;
\end{aligned}
$$

the last two lines indicate again jumps over the interface points. With appropriate evaluation points $\zeta_{j}(x, t)$ between $u(x, t)$ and $u_{+}(x, t)$ we may write this as

$$
\begin{aligned}
& \partial_{t} v+\partial_{x}\left(f_{u}\left(., \zeta_{1}\right) v-g_{u}\left(., \zeta_{2}\right) v \partial_{x} u_{+}-g(., u) \partial_{x} v\right)=0, \\
& \left\{\partial_{u} p_{c}\left(., \zeta_{3}\right) v\right\}(k \varepsilon+0)=\left\{\partial_{u} p_{c}\left(., \zeta_{4}\right) v\right\}(k \varepsilon-0), \\
& \left\{f_{u}\left(., \zeta_{5}\right) v-g_{u}\left(., \zeta_{6}\right) v \partial_{x} u_{+}-g(., u) \partial_{x} v\right\}(k \varepsilon+0) \\
& \quad=\left\{f_{u}\left(., \zeta_{7}\right) v-g_{u}\left(., \zeta_{8}\right) v \partial_{x} u_{+}-g(., u) \partial_{x} v\right\}(k \varepsilon-0)
\end{aligned}
$$

for $x=k \varepsilon$ with $k \in \mathbb{Z}$. Starting from this system for $v$, the nonnegativity of $v$ follows as in part 1 .

LEMma 4 (bounds for averages of $u^{\varepsilon}$ ). Let $u^{\varepsilon}$ be a family of entropy solutions of (1.11) as above.

1. Lower bound for averages. There exists $c \in \mathbb{R}$ such that for all $\varepsilon>0$ and all $k \in \mathbb{Z}$ with $(2 k \varepsilon-\varepsilon, 2 k \varepsilon+\varepsilon) \subset(0, L)$, there holds

$$
\int_{2 k \varepsilon-\varepsilon}^{2 k \varepsilon+\varepsilon} u^{\varepsilon}(., t) \geq \varepsilon\left(u^{*}-c \varepsilon\right) .
$$

2. Upper bound for averages. For every $\rho>0$ there exist $\tau>0, \delta>0$, and $\varepsilon_{0}>0$ such that for all $\varepsilon \in\left(0, \varepsilon_{0}\right), k \in \mathbb{Z}$ with $(2 k \varepsilon-\varepsilon, 2 k \varepsilon+\varepsilon) \subset(0, L)$, and $t>\tau \varepsilon^{2}$,

$$
u^{\varepsilon}\left(2 k \varepsilon+\varepsilon-0, t-\tau \varepsilon^{2}\right) \leq \delta \quad \Rightarrow \quad \int_{2 k \varepsilon-\varepsilon}^{2 k \varepsilon+\varepsilon} u^{\varepsilon}(., t) \leq \varepsilon\left(u^{*}+\rho\right) .
$$

Proof. We consider again approximate solutions $u_{\eta}^{\varepsilon}$ from the definition of entropy solutions.

Proof of part 1 . We approximate additionally the boundary condition at the left boundary by the artificial condition $u_{\eta}^{\varepsilon}(0, t)=\delta$. The subsolutions $U^{\varepsilon}$ of Lemma 2 satisfy $U^{\varepsilon} \geq u^{*}-O(\varepsilon)$ on $\Gamma_{+}^{\varepsilon}$, independent of $\delta>0$, such that $u_{\eta}^{\varepsilon} \geq U^{\varepsilon}$ provides (2.4).

Copyright $@$ by SIAM. Unauthorized reproduction of this article is prohibited. 
Proof of part 2. We assume the contrary. Then, for some $\rho>0$, for arbitrary $\tau>0, \delta>0$, there exists a sequence $\varepsilon_{m} \rightarrow 0$ such that inequality (2.5) fails to hold for some $k$ and $t$. Now let $\rho>0$ be such a value. Below we give an explicit choice of $\tau$ and $\delta$ that leads to a contradiction. We study now sequences of points $k_{m}$ and time instances $t_{m} \geq \tau \varepsilon_{m}^{2}$ at which (2.5) fails for the sequence $u^{\varepsilon_{m}}$, which we continue up to time $2 T$. We define the rescaled solutions

$$
\begin{aligned}
\tilde{U}^{m} & :(-1,1) \times\left(0, T / \varepsilon_{m}^{2}\right) \rightarrow \mathbb{R}, \\
\tilde{U}^{m}(y, s) & :=u^{\varepsilon_{m}}\left(2 k_{m} \varepsilon_{m}+\varepsilon_{m} y, t_{m}-\tau \varepsilon_{m}^{2}+\varepsilon_{m}^{2} s\right)
\end{aligned}
$$

and recall that we assume the failure of (2.5), that is,

$$
\tilde{U}^{m}(1-0, s=0) \leq \delta, \quad \int_{-1}^{1} \tilde{U}^{m}(y, \tau) d y>u^{*}+\rho .
$$

We now construct a function $U^{m}$ which serves as an upper bound for $\tilde{U}^{m}$. We define $U^{m}$ as the solution of the system

$$
\begin{aligned}
& \partial_{s} U^{m}+\partial_{y}\left(\varepsilon_{m} f\left(U^{m}\right)-g\left(U^{m}\right) \partial_{y} U^{m}\right)=0 \text { on }(-1,1) \backslash\{0\}, \\
& U^{m}(1-0)=\delta, \quad U^{m}(-1+0)=\left(p_{c}{ }^{+}\right)^{-1}\left(p_{c}{ }^{-}(\delta)\right), \\
& p_{c}{ }^{+}\left(U^{m}(0-0)\right)=p_{c}{ }^{-}\left(U^{m}(0+0)\right), \\
& {\left[\varepsilon_{m} f\left(U^{m}\right)-g\left(U^{m}\right) \partial_{y} U^{m}\right](0-0)=\left[\varepsilon_{m} f\left(U^{m}\right)-g\left(U^{m}\right) \partial_{y} U^{m}\right](0+0),}
\end{aligned}
$$

but now augmented with the initial condition $p_{c}\left(U^{m}(., s=0)\right) \equiv p_{\max }$. As in the above proofs, exploiting $\tilde{U}^{m}(1-0,0) \leq \delta$ and the monotonicity of $\tilde{U}^{m}$, one derives the comparison principle $\tilde{U}^{m} \leq U^{m}$. The limit $U^{\infty}:=\lim _{m \rightarrow \infty} U^{m}$ exists and solves the above system with $\varepsilon_{m}$ replaced by 0 . The solution $U^{\infty}$ approaches, as $s \rightarrow \infty$, the stationary solution

$$
\bar{U}^{\infty}(y)= \begin{cases}\left(p_{c}{ }^{+}\right)^{-1}\left(p_{c}{ }^{-}(\delta)\right) & \text { for } y \in(-1,0), \\ \delta & \text { for } y \in(0,1) .\end{cases}
$$

We can now derive a contradiction. Given $\rho>0$, we choose $\delta>0$ such that $\int_{-1}^{1} \bar{U}^{\infty}(y) d y<u^{*}+\rho / 3$. We then choose a time instance $\tau>0$ such that $\int_{-1}^{1}\left(U^{\infty}(y, \tau)\right.$ $\left.-\bar{U}^{\infty}(y)\right) d y<\rho / 3$. With these choices we have

$$
u^{*}+\rho<\int_{-1}^{1} \tilde{U}^{m}(y, \tau) d y \leq \int_{-1}^{1} U^{m}(y, \tau) d y \rightarrow \int_{-1}^{1} U^{\infty}(y, \tau) d y \leq u^{*}+2 \rho / 3,
$$

a contradiction.

3. Homogenization for a positive saturation. We next define the nonlinear flux function $\mathcal{F}\left(u^{0}, v^{0}\right)$ that maps an average oil saturation $u^{0}$ with an average slope $v^{0}$ to the effective flux. The continuity of the capillary pressure imposes a restriction on the values of $u^{0}$. Let $U \in[0,1]$ solve $p_{c}{ }^{-}(U)=p_{c}{ }^{+}(1)$. Then, with $u_{\max }^{0}:=(1+U) / 2$, the flux function is a map

$$
\mathcal{F}:\left[0, u_{\text {max }}^{0}\right] \times \mathbb{R} \mapsto \mathbb{R},\left(u^{0}, v^{0}\right) \mapsto \mathcal{F}\left(u^{0}, v^{0}\right) .
$$

We set $\mathcal{F}\left(u^{0}, v^{0}\right):=0$ for all $\left(u^{0}, v^{0}\right)$ with $u^{0} \leq u^{*} / 2$ and construct $\mathcal{F}$ for other values with the help of nonlinear equations. For $\left(u^{0}, v^{0}\right) \in\left(u^{*} / 2, u_{\text {max }}^{0}\right] \times \mathbb{R}$, the following 
system determines $\left(u_{+}, u_{-}\right) \in[0,1]^{2}$, representing typical values of $u^{\varepsilon}$ in $\Gamma_{ \pm}^{\varepsilon}$ :

$$
\begin{aligned}
& u_{+}+u_{-}=2 u^{0}, \\
& p_{c}{ }^{+}\left(u_{+}\right)=p_{c}{ }^{-}\left(u_{-}\right) .
\end{aligned}
$$

The monotonicity of $p_{c}{ }^{ \pm}$assures the unique solvability of (3.1)-(3.2). We introduce auxiliary real numbers $u_{+, x}$ and $u_{-, x}$ that will describe the average slope of $u_{+}$and $u_{-}$on a macroscopic scale. They are determined by

$$
\begin{aligned}
u_{+, x}+u_{-, x} & =2 v^{0}, \\
\partial_{u} p_{c}^{+}\left(u_{+}\right) u_{+, x} & =\partial_{u} p_{c}^{-}\left(u_{-}\right) u_{-, x} .
\end{aligned}
$$

This linear system has a unique solution $u_{ \pm, x}$ that depends linearly on $v_{0}$. We note that, for $v^{0} \geq 0$, the average slope satisfies $0 \leq u_{ \pm, x} \leq 2 v^{0}$. We next introduce the pair $\left(v_{+}, v_{-}\right) \in \mathbb{R}^{2}$ which describes the typical derivatives of $u^{\varepsilon}$ inside a single interval of $\Gamma_{ \pm}^{\varepsilon}$. They are determined by

$$
\begin{aligned}
f^{+}\left(u_{+}\right)-g^{+}\left(u_{+}\right) v_{+} & =f^{-}\left(u_{-}\right)-g^{-}\left(u_{-}\right) v_{-}, \\
\partial_{u} p_{c}{ }^{+}\left(u_{+}\right) v_{+}+\partial_{u} p_{c}^{-}\left(u_{-}\right) v_{-} & =\partial_{u} p_{c}^{+}\left(u_{+}\right) u_{+, x}+\partial_{u} p_{c}^{-}\left(u_{-}\right) u_{-, x} .
\end{aligned}
$$

The unique solution $v_{ \pm}$depends in an affine way on $u_{ \pm, x}$. We now define the effective flux function $\mathcal{F}$ as

$$
\mathcal{F}\left(u^{0}, v^{0}\right):=f^{+}\left(u_{+}\right)-g^{+}\left(u_{+}\right) v_{+},
$$

where $\left(u_{+}, v_{+}\right)$is determined by the system (3.1)-(3.6) of nonlinear equations. For fixed $u \in\left[0, u_{\text {max }}^{0}\right]$, the map $\mathcal{F}(u,$.$) is affine. We may therefore also write \mathcal{F}$ in the form

$$
\mathcal{F}\left(u^{0}, v^{0}\right)=\mathcal{F}_{0}\left(u^{0}\right)-\mathcal{D}\left(u^{0}\right) v^{0} .
$$

A flux function of this form appears also in [10] and [9]. We note that $\mathcal{F}$ is continuous: For $u^{0}=u^{*} / 2$, the solution of system (3.1)-(3.2) is $u_{-}=0$ and $u_{+}=u^{*}$, and hence $f^{-}\left(u_{-}\right)=g^{-}\left(u_{-}\right)=0$, and $(3.5)$ yields $\mathcal{F}\left(u^{0}, v^{0}\right)=0$.

Proposition 1 (homogenization). Let $G=(a, b) \times\left(0, t_{0}\right)$ be a subdomain of $\Omega_{T}=(0, L) \times(0, T), \delta, \varepsilon_{0}>0$ positive real numbers, and $u^{\varepsilon}$ a family of solutions of (1.11) with

$$
\begin{aligned}
u^{\varepsilon} \geq \delta & \text { on } G \forall \varepsilon \leq \varepsilon_{0}, \\
u^{\varepsilon}-u^{0}, \quad F^{\varepsilon} \rightarrow F^{0} & \text { weakly in } L^{2}(G) .
\end{aligned}
$$

Then $u^{0} \in L^{2}(G)$ solves $\partial_{t} u^{0}+\partial_{x} F^{0}=0$ in the distributional sense on $G$ and has a space derivative $\partial_{x} u^{0} \in L^{2}(G)$, and, with $\mathcal{F}$ from (3.7), the following flux relation holds almost everywhere:

$$
F^{0}=\mathcal{F}\left(u^{0}, \partial_{x} u^{0}\right)
$$

Proof. The distributional conservation law is satisfied by the weak convergences. Exploiting the strictly positive diffusivity, (2.1) provides an estimate for the regular part of the derivative, and $\partial_{x} u^{\varepsilon}(x, t) \mathbf{1}_{\Gamma^{\varepsilon}}(x)$ is bounded in $L^{2}(G)$. In the subsequent proof we will define various two-scale limits for the above functions. For them we derive the relations (3.1)-(3.6), $F^{0}=f^{+}\left(u_{+}\right)-g^{+}\left(u_{+}\right) v_{+}$, and $\partial_{x} u^{0}=v^{0} \in L^{2}(G)$. With these verifications, the proof is complete. 
Step 1. Two-scale limits and (3.1). The uniform $L^{2}(G)$-bounds allow us to consider the two-scale limits

$$
\begin{aligned}
u^{\varepsilon} & \rightarrow u_{0}(x, t, y) \text { two-scale, } \\
\partial_{x} u^{\varepsilon} \mathbf{1}_{\Gamma^{\varepsilon}}(x) & -v_{0}(x, t, y) \text { two-scale. }
\end{aligned}
$$

The $L^{2}(G)$-estimate for $\partial_{x} u^{\varepsilon}$ immediately implies that $u_{0}$ is independent of $y$ on the sets $(0,1)$ and $(1,2)$. Indeed, let $y \mapsto \varphi(y)$ be smooth with support contained in one of the two sets. We find, for $\Phi^{\varepsilon}(x)=\varepsilon \psi(x) \varphi(x / \varepsilon)$,

$$
0 \leftarrow \int_{G} \partial_{x} u^{\varepsilon} \Phi^{\varepsilon}=\int_{G} u^{\varepsilon} \partial_{x} \Phi^{\varepsilon} \rightarrow \int_{G} \int_{0}^{2} u_{0}(x, t, y) \psi(x) \partial_{y} \varphi(y) d y d x d t .
$$

We conclude that $u_{0}$ has the special form

$$
u_{0}(x, t, y)=u_{-}(x, t) \mathbf{1}_{(0,1)}(y)+u_{+}(x, t) \mathbf{1}_{(1,2)}(y) .
$$

The weak limit $u^{0}$ of the sequence $u^{\varepsilon}$ coincides with the $y$-average of $u_{0}$; hence (3.11) implies relation (3.1).

We claim that also $v_{0}$ is piecewise constant. To see this, we use the test function $\Phi^{\varepsilon}(x, t)=\varepsilon \psi(x, t) \varphi(x / \varepsilon)$ with $\psi \in C_{0}^{\infty}(G)$ and exploit the equation. We assume here that $\varphi$ is supported in $(1,2)$. We will verify the limit of the second line (marked with an exclamation mark) in the next step of the proof:

$$
\begin{aligned}
& 0 \leftarrow \int_{G} \partial_{t} u^{\varepsilon} \Phi^{\varepsilon}=\int_{G} f^{\varepsilon}\left(u^{\varepsilon}\right) \partial_{x} \Phi^{\varepsilon}-\int_{G} g^{\varepsilon}\left(u^{\varepsilon}\right) \partial_{x} u^{\varepsilon} \partial_{x} \Phi^{\varepsilon} \\
& \stackrel{!}{\rightarrow} \int_{G} \int_{1}^{2} f^{+}\left(u_{+}(x, t)\right) \psi(x, t) \partial_{y} \varphi(y) d y d x d t \\
& \quad-\int_{G} \int_{1}^{2} g^{+}\left(u_{+}(x, t)\right) v_{0}(x, t, y) \psi(x, t) \partial_{y} \varphi(y) d y d x d t .
\end{aligned}
$$

The first integral vanishes since $\varphi$ is compactly supported in $(1,2)$ and we conclude that $v_{0}$ is independent of $y$, since $u_{+}$is positive by the lower bound on $u^{\varepsilon}$. We can perform the same calculations with $\varphi$ supported in $(0,1)$ to find the same equality with + replaced by - ,

$$
v_{0}(x, t, y)=v_{-}(x, t) \mathbf{1}_{(0,1)}(y)+v_{+}(x, t) \mathbf{1}_{(1,2)}(y) .
$$

In particular, the quantities $u_{ \pm}=u_{ \pm}(x)$ and $v_{ \pm}=v_{ \pm}(x)$ that appear in (3.1)-(3.6) are now defined. For brevity, we will often suppress the dependence on $t$ in the following.

Step 2. Compactness. To abbreviate notation we write $I=(a, b)$ for the spatial interval and set $\mathbf{1}_{k}:=\mathbf{1}_{(2 k \varepsilon, 2 k \varepsilon+2 \varepsilon)}, \mathbf{1}_{k}^{-}:=\mathbf{1}_{(2 k \varepsilon, 2 k \varepsilon+\varepsilon)}$, and $\mathbf{1}_{k}^{+}:=\mathbf{1}_{(2 k \varepsilon+\varepsilon, 2 k \varepsilon+2 \varepsilon)}$. We furthermore set $\mathbf{1}_{+}^{\varepsilon}:=\sum_{k} \mathbf{1}_{k}^{+}$and $\mathbf{1}_{-}^{\varepsilon}:=\sum_{k} \mathbf{1}_{k}^{-}$. Our aim in this step of the proof is the following result. Let $h:[0,1] \rightarrow \mathbb{R}$ be a continuous function. Then

$$
h\left(u^{\varepsilon}(x)\right) \mathbf{1}_{-}^{\varepsilon}(x)-h\left(u_{-}(x)\right) \mathbf{1}_{-}^{\varepsilon}(x) \rightarrow 0 \text { strongly in } L^{2}(G),
$$

and likewise for - replaced by + . We note that this result justifies, with $h=g^{+}$, the convergence in (3.12). We emphasize that (3.14) is not a consequence of the previous results. For its proof we must control variations of $u^{\varepsilon}$ on points in $2 \mathbb{Z} \varepsilon$. Loosely speaking, it must jump down in $2 k \varepsilon+2 \varepsilon$ as much as it jumped up in $2 k \varepsilon+\varepsilon$.

Copyright $@$ by SIAM. Unauthorized reproduction of this article is prohibited. 
In order to derive (3.14) we consider the capillary pressure function $P^{\varepsilon}(x)=$ $p_{c}{ }^{\varepsilon}\left(x, u^{\varepsilon}(x)\right)$. This function has no jumps across interfaces, and hence the spatial derivative has no singular parts. On $\Gamma^{\varepsilon}$ we have the estimate $\left|\partial_{x} P^{\varepsilon}\right| \leq C\left|\partial_{x} u^{\varepsilon}\right|$, and therefore a uniform estimate for $P^{\varepsilon} \in L^{2}\left(\left(0, t_{0}\right), H^{1}(I)\right)$.

We have seen in Lemma 3 that $t \mapsto u^{\varepsilon}(x, t)$ is monotone for almost every $x \in I$. By the monotonicity of $p_{c}{ }^{ \pm}$, this implies the monotonicity of $t \mapsto P^{\varepsilon}(x, t)$. For the strong solutions of the strictly parabolic equations of the proposition we therefore have $\left|\partial_{t} P^{\varepsilon}\right|=-\partial_{t} P^{\varepsilon}$, and an integration yields $\left\|\partial_{t} P^{\varepsilon}\right\|_{L^{1}(G)} \leq L\left\|P^{\varepsilon}\right\|_{L^{\infty}(G)}$, which is uniformly bounded. The spatial and the temporal regularity together provide the boundedness of $P^{\varepsilon}$ in $W^{1,1}(G)$, hence the precompactness of $P^{\varepsilon}$ in $L^{1}(G)$. Exploiting once more the uniform bound in $L^{\infty}(G)$, we find a subsequence that converges strongly in $L^{2}(G)$ and almost everywhere in $G$ to a limit $P^{0} \in L^{2}(G)$.

The convergence almost everywhere can be exploited to conclude the strong convergence of $u^{\varepsilon}$ as claimed in (3.14). Since $\left(P^{\varepsilon}-P^{0}\right) \mathbf{1}_{-}^{\varepsilon} \rightarrow 0$ pointwise, also

$$
u^{\varepsilon} \mathbf{1}_{-}^{\varepsilon}-\left(p_{c}^{-}\right)^{-1}\left(P^{0}\right) \mathbf{1}_{-}^{\varepsilon} \rightarrow 0
$$

pointwise almost everywhere and, by the uniform boundedness, also strongly in $L^{2}(G)$. In order to identify the limit function we recall that $u^{\varepsilon} \mathbf{1}_{-}^{\varepsilon} \rightarrow u_{-}(x) \mathbf{1}_{(0,1)}(y)$ in the sense of two-scale convergence. On the other hand, again in the sense of two-scale convergence, $\left(p_{c}{ }^{-}\right)^{-1}\left(P^{0}(x)\right) \mathbf{1}_{-}^{\varepsilon}(x) \rightarrow\left(p_{c}{ }^{-}\right)^{-1}\left(P^{0}\right)(x) \mathbf{1}_{(0,1)}(y)$, and hence $\left(p_{c}{ }^{-}\right)^{-1}\left(P^{0}\right)=$ $u_{-}$.

We can now also apply a nonlinear continuous function $h$ to both expressions in $(3.15)$ and find $h\left(u^{\varepsilon}\right) \mathbf{1}_{-}^{\varepsilon}-h\left(u_{-}\right) \mathbf{1}_{-}^{\varepsilon} \rightarrow 0$ pointwise almost everywhere. By the uniform bounds for $u^{\varepsilon}$, this provides (3.14).

Step 3. Derivation of the continuity conditions (3.2) and (3.5) and the flux equality. With the help of (3.14) it is not difficult to derive the continuity conditions. The strong convergence $P^{\varepsilon} \rightarrow P^{0}$ in $L^{2}(G)$, together with $P^{0} \in L^{2}\left(\left(0, t_{0}\right), H^{1}(I)\right)$, implies

$$
p_{c}^{\varepsilon}\left(u^{\varepsilon}\right) \mathbf{1}_{+}^{\varepsilon}-p_{c}^{\varepsilon}\left(u^{\varepsilon}\right) \mathbf{1}_{-}^{\varepsilon}=P^{\varepsilon} \mathbf{1}_{+}^{\varepsilon}-P^{\varepsilon} \mathbf{1}_{-}^{\varepsilon} \rightarrow 0 \text { in } L^{2}(G) .
$$

On the other hand, by (3.14) and the two-scale convergences,

$$
\begin{aligned}
p_{c}^{\varepsilon}\left(u^{\varepsilon}\right) \mathbf{1}_{+}^{\varepsilon}-p_{c}^{\varepsilon}\left(u^{\varepsilon}\right) \mathbf{1}_{-}^{\varepsilon} & =p_{c}{ }^{+}\left(u^{\varepsilon}\right) \mathbf{1}_{+}^{\varepsilon}-p_{c}{ }^{-}\left(u^{\varepsilon}\right) \mathbf{1}_{-}^{\varepsilon} \\
& =p_{c}{ }^{+}\left(u_{+}\right) \mathbf{1}_{+}^{\varepsilon}-p_{c}{ }^{-}\left(u_{-}\right) \mathbf{1}_{-}^{\varepsilon}+o(1) \\
& \rightarrow \frac{1}{2}\left(p_{c}{ }^{+}\left(u_{+}\right)-p_{c}{ }^{-}\left(u_{-}\right)\right) .
\end{aligned}
$$

Comparison of the two limits yields (3.2).

For the derivation of $(3.5)$ we consider a test function $\varphi \in C_{0}^{\infty}((0,2), \mathbb{R})$, and $\Phi^{\varepsilon}(x)=\varepsilon \psi(x, t) \varphi(x / \varepsilon)$ as above. Exploiting (3.14) we find

$$
\begin{aligned}
0 & \leftarrow \int_{G} \partial_{t} u^{\varepsilon} \Phi^{\varepsilon}=\int_{G} f\left(u^{\varepsilon}\right) \partial_{x} \Phi^{\varepsilon}-\int_{G} g^{\varepsilon}\left(u^{\varepsilon}\right) \partial_{x} u^{\varepsilon} \partial_{x} \Phi^{\varepsilon} \\
\rightarrow & \int_{G} \int_{0}^{1} f^{-}\left(u_{-}\right) \psi \partial_{y} \varphi d y+\int_{G} \int_{1}^{2} f^{+}\left(u_{+}\right) \psi \partial_{y} \varphi d y \\
& -\int_{G} \int_{0}^{1} g^{-}\left(u_{-}\right) v_{-} \psi \partial_{y} \varphi d y-\int_{G} \int_{1}^{2} g^{+}\left(u_{+}\right) v_{+} \psi \partial_{y} \varphi d y \\
= & \int_{G} \psi\left[f^{-}\left(u_{-}\right)-f^{+}\left(u_{+}\right)\right] \varphi(1)-\int_{G} \psi\left[g^{-}\left(u_{-}\right) v_{-}-g^{+}\left(u_{+}\right) v_{+}\right] \varphi(1) .
\end{aligned}
$$

Copyright (c) by SIAM. Unauthorized reproduction of this article is prohibited. 
Since $\psi$ was arbitrary, this yields (3.5). In order to derive the flux equality, we exploit once more (3.14) and calculate

$$
\begin{aligned}
F^{\varepsilon} & =f^{\varepsilon}\left(u^{\varepsilon}\right)-g^{\varepsilon}\left(u^{\varepsilon}\right) \partial_{x} u^{\varepsilon} \\
& =\left[f^{+}\left(u^{\varepsilon}\right)-g^{+}\left(u^{\varepsilon}\right) \partial_{x} u^{\varepsilon}\right] \mathbf{1}_{+}^{\varepsilon}+\left[f^{-}\left(u^{\varepsilon}\right)-g^{-}\left(u^{\varepsilon}\right) \partial_{x} u^{\varepsilon}\right] \mathbf{1}_{-}^{\varepsilon} \\
& -\frac{1}{2}\left[f^{+}\left(u_{+}\right)-g^{+}\left(u_{+}\right) v_{+}\right]+\frac{1}{2}\left[f^{-}\left(u_{-}\right)-g^{-}\left(u_{-}\right) v_{-}\right],
\end{aligned}
$$

which, because of (3.5), is the result for $F^{0}$.

Step 4 . The quantities $u_{ \pm, x}$ and relation (3.3). Our aim is to derive $u_{-, x}:=$ $\partial_{x} u_{-} \in L^{2}(G)$. Loosely speaking, we need an estimate for the oscillations of $u^{\varepsilon}$ on $\Gamma_{-}^{\varepsilon}$. Such an estimate is the consequence of the corresponding estimate for the capillary pressures $P^{\varepsilon}$. Our construction serves also as a preparation for Step 5 .

We introduce a function $\hat{P}^{\varepsilon}$ as a piecewise affine approximation of $P^{\varepsilon}$,

$$
\begin{array}{cc}
\hat{P}^{\varepsilon}(2 k \varepsilon)=\frac{1}{\varepsilon} \int_{2 k \varepsilon}^{2 k \varepsilon+\varepsilon} P^{\varepsilon} & \forall k, \\
\hat{P}^{\varepsilon} \text { affine on }(2 k \varepsilon, 2 k \varepsilon+2 \varepsilon) & \forall k .
\end{array}
$$

Exploiting the $L^{2}\left(\left(0, t_{0}\right), H^{1}(I)\right)$-regularity of $P^{\varepsilon}$ we find $\hat{P}^{\varepsilon} \rightarrow P^{0}$. Furthermore, as a projection of $P^{\varepsilon}$ onto the space of piecewise affine functions, the projections $\hat{P}^{\varepsilon}$ are again bounded in $L^{2}\left(\left(0, t_{0}\right), H^{1}(\Omega)\right)$. Choosing a subsequence, we may assume

$$
\partial_{x} \hat{P}^{\varepsilon}=\sum_{k} \mathbf{1}_{k} \frac{1}{2 \varepsilon^{2}} \int_{2 k \varepsilon}^{2 k \varepsilon+\varepsilon}\left[P^{\varepsilon}(.+2 \varepsilon)-P^{\varepsilon}(.)\right] \rightarrow \partial_{x} P^{0} \text { in } L^{2}(G) .
$$

We can now relate the function $\hat{P}^{\varepsilon}$ with a piecewise linear function $\hat{u}^{\varepsilon}$ that approximates $u_{-}$,

$$
\begin{array}{cc}
\hat{u}^{\varepsilon}(2 k \varepsilon)=\frac{1}{\varepsilon} \int_{2 k \varepsilon}^{2 k \varepsilon+\varepsilon} u^{\varepsilon} & \forall k, \\
\hat{u}^{\varepsilon} \text { affine on }(2 k \varepsilon, 2 k \varepsilon+2 \varepsilon) & \forall k
\end{array}
$$

with derivative

$$
\partial_{x} \hat{u}^{\varepsilon}=\sum_{k} \mathbf{1}_{k} \frac{1}{2 \varepsilon^{2}} \int_{2 k \varepsilon}^{2 k \varepsilon+\varepsilon}\left[u^{\varepsilon}(.+2 \varepsilon)-u^{\varepsilon}(.)\right] .
$$

We claim that the sequence $\partial_{x} \hat{u}^{\varepsilon}$ is uniformly bounded in $L^{2}(G)$. Our aim is to compare $\partial_{x} \hat{P}^{\varepsilon}$ with $\partial_{u} p_{c}{ }^{-}\left(u_{-}\right) \cdot \partial_{x} \hat{u}^{\varepsilon}$. To this end we write the pressure derivative with the fundamental theorem and the function $\xi(x, \lambda):=\lambda u^{\varepsilon}(x+2 \varepsilon)+(1-\lambda) u^{\varepsilon}(x)$ as

$$
\partial_{x} \hat{P}^{\varepsilon}=\sum_{k} \mathbf{1}_{k} \frac{1}{2 \varepsilon^{2}} \int_{2 k \varepsilon}^{2 k \varepsilon+\varepsilon}\left\{\int_{0}^{1} \partial_{u} p_{c}{ }^{-}(\xi(x, \lambda)) d \lambda\right\}\left[u^{\varepsilon}(x+2 \varepsilon)-u^{\varepsilon}(x)\right] d x .
$$

The nonnegativity of the integrand provided by Lemma 3 and the lower bound for $\partial_{u} p_{c}{ }^{-}$imply a uniform bound for $\partial_{x} \hat{u}^{\varepsilon} \in L^{2}(G)$. In particular, we may assume that $\hat{u}^{\varepsilon}$ converges strongly in $L^{2}(G)$; the limit is easily identified with the weak limit $u_{-}$.

Copyright $@$ by SIAM. Unauthorized reproduction of this article is prohibited. 
Furthermore, choosing a subsequence, we may assume that $\partial_{x} \hat{u}^{\varepsilon}$ converges weakly in $L^{2}(G)$. Denoting the limit function by $u_{-, x}$ we have

$$
\partial_{x} \hat{u}^{\varepsilon}-u_{-, x}:=\partial_{x} u_{-} \text {in } L^{2}(G) .
$$

In a similar way one constructs functions $\tilde{u}^{\varepsilon}$ that approximate $u_{+}$with $\partial_{x} \tilde{u}^{\varepsilon}$ bounded in $L^{2}(G)$. We may assume that also this sequence converges weakly, $\partial_{x} \tilde{u}^{\varepsilon} \rightarrow u_{+, x}:=$ $\partial_{x} u_{+}$in $L^{2}(G)$.

The weak convergences $\hat{u}^{\varepsilon} \rightarrow u_{-}$and $\tilde{u}^{\varepsilon} \rightarrow u_{+}$, together with (3.1), imply $\hat{u}^{\varepsilon}+$ $\tilde{u}^{\varepsilon}-2 u^{0}$. Then the distributional derivatives converge as well, and we conclude (3.3) with $v^{0}=\partial_{x} u^{0} \in L^{2}(G)$.

Step 5. Derivation of (3.4) and (3.6). We have seen that the capillary pressure functions $P^{\varepsilon}$ are bounded in $L^{2}\left(\left(0, t_{0}\right), H^{1}(I)\right)$ and that we may therefore assume $\partial_{x} P^{\varepsilon} \rightarrow \partial_{x} P^{0}$ in $L^{2}(G)$. In this last step of the proof we calculate the derivative $\partial_{x} P^{0}$ in three different ways.

The most direct approach is to calculate with the chain rule, exploiting (3.14) in the second equality,

$$
\begin{aligned}
\partial_{x} P^{0}-\partial_{x} P^{\varepsilon} & =\partial_{u} p_{c}{ }^{-}\left(u^{\varepsilon}\right) \partial_{x} u^{\varepsilon} \mathbf{1}_{-}^{\varepsilon}+\partial_{u} p_{c}{ }^{+}\left(u^{\varepsilon}\right) \partial_{x} u^{\varepsilon} \mathbf{1}_{+}^{\varepsilon} \\
& =\partial_{u} p_{c}{ }^{-}\left(u_{-}\right) \partial_{x} u^{\varepsilon} \mathbf{1}_{-}^{\varepsilon}+\partial_{u} p_{c}{ }^{+}\left(u_{+}\right) \partial_{x} u^{\varepsilon} \mathbf{1}_{+}^{\varepsilon}+o(1) \\
& -\frac{1}{2} \partial_{u} p_{c}{ }^{-}\left(u_{-}\right) v_{-}+\frac{1}{2} \partial_{u} p_{c}{ }^{+}\left(u_{+}\right) v_{+}
\end{aligned}
$$

We will now calculate $\partial_{x} P^{0}$ in a different way. We introduce the function $P^{*, \varepsilon}:=$ $p_{c}{ }^{-}\left(\hat{u}^{\varepsilon}\right)$. The monotonicity of $\hat{u}^{\varepsilon}$ in $t$ implies a compactness and allows us to assume the strong and the pointwise almost everywhere convergence $\hat{u}^{\varepsilon} \rightarrow u_{-}$. We calculate with the chain rule

$$
\partial_{x} P^{*, \varepsilon}=\partial_{u} p_{c}{ }^{-}\left(\hat{u}^{\varepsilon}\right) \cdot \partial_{x} \hat{u}^{\varepsilon} \rightarrow \partial_{u} p_{c}{ }^{-}\left(u_{-}\right) \cdot u_{-, x} \text { in } L^{2}(G) .
$$

On the other hand, $P^{*, \varepsilon}=p_{c}{ }^{-}\left(\hat{u}^{\varepsilon}\right) \rightarrow p_{c}{ }^{-}\left(u_{-}\right)=P^{0}$, and therefore the distributional limits coincide,

$$
\partial_{x} P^{0}=\partial_{u} p_{c}{ }^{-}\left(u_{-}\right) \cdot u_{-, x} .
$$

The above calculation can also be performed with averages over the set $\Gamma_{\varepsilon}^{+}$and with the function $p_{c}^{+}$. We find the analogous formula $\partial_{x} P^{0}=\partial_{u} p_{c}^{+}\left(u_{+}\right) \cdot u_{+, x}$ and thus (3.4). In (3.6) we use the symmetric version

$$
\partial_{x} P^{0}=\frac{1}{2} \partial_{u} p_{c}{ }^{+}\left(u_{+}\right) u_{+, x}+\frac{1}{2} \partial_{u} p_{c}{ }^{-}\left(u_{-}\right) u_{-, x} .
$$

From our first calculation of $\partial_{x} P^{0}$ we see that the weighted average of $u_{+, x}$ and $u_{-, x}$ coincides with the weighted average of $v_{+}$and $v_{-}$, as claimed in (3.6).

As a preparation for the investigation of the interface condition in the free boundary value problem, we investigate the regularity of solutions in the region of strictly positive saturation.

Lemma 5 (Hölder's estimate). We consider a family of entropy solutions $u^{\varepsilon}$ and fix positive numbers $C_{0}$ and $\delta$. We assume that $t \in(0, T)$ is a time instance of bounded energy in the sense that

$$
\int_{\Gamma^{\varepsilon}}\left|\partial_{x} u^{\varepsilon}(., t)\right|^{2} \mathbf{1}_{\left\{u^{\varepsilon} \geq \delta / 2\right\}} \leq C_{0}^{2}
$$

Copyright $@$ by SIAM. Unauthorized reproduction of this article is prohibited. 
Then there exist a constant $C_{L}=C_{L}(\delta)$, independent of $C_{0}$ and $\varepsilon$, and a constant $\varepsilon_{0}=\varepsilon_{0}\left(\delta, C_{0}\right)$ such that the following hold:

1. Let $a=2 k \varepsilon+\varepsilon \in(0, L)$ with $k \in \mathbb{Z}$ and $u^{\varepsilon}(a-0, t) \geq \delta$, and let $b \in 2 \mathbb{Z} \varepsilon+\varepsilon$, $b>a$. Then

$$
\left|u^{\varepsilon}(b-0, t)-u^{\varepsilon}(a-0, t)\right| \leq C_{L} C_{0} \sqrt{|b-a|} .
$$

2. For all $\varepsilon \leq \varepsilon_{0}\left(\delta, C_{0}\right)$ there holds

$$
u^{\varepsilon}(2 k \varepsilon+\varepsilon-0, t) \geq \delta \quad \Rightarrow \quad u^{\varepsilon}(2 k \varepsilon-\varepsilon-0, t) \geq \delta / 2 .
$$

Proof. Cellwise estimate and (3.17). The monotonicity of Lemma 3, together with the lower bound of Lemma 2, implies $u^{\varepsilon} \geq \delta$ on $(a, L) \times\left(0, t_{0}\right)$, and hence (3.16) provides an $L^{2}\left(\Gamma^{\varepsilon}\right)$-bound for the spatial derivative. We claim that locally, across a single interval $(2 k \varepsilon+\varepsilon, 2 k \varepsilon+3 \varepsilon)$, we can control differences of the $u^{\varepsilon}$ values by the integral of the derivative. Indeed, with the variables

$$
\begin{array}{ll}
y_{0}:=u^{\varepsilon}(2 k \varepsilon+\varepsilon-0, t), & y_{1}:=u^{\varepsilon}(2 k \varepsilon+2 \varepsilon+0, t), \quad y_{2}:=u^{\varepsilon}(2 k \varepsilon+3 \varepsilon-0, t), \\
z_{0}:=u^{\varepsilon}(2 k \varepsilon+\varepsilon+0, t), & z_{1}:=u^{\varepsilon}(2 k \varepsilon+2 \varepsilon-0, t),
\end{array}
$$

we have the relations

$$
\begin{aligned}
& y_{2}-y_{1}=\int_{2 k \varepsilon+2 \varepsilon}^{2 k \varepsilon+3 \varepsilon} \partial_{x} u^{\varepsilon}(., t)=: \Delta_{1}, \\
& z_{1}-z_{0}=\int_{2 k \varepsilon+\varepsilon}^{2 k \varepsilon+2 \varepsilon} \partial_{x} u^{\varepsilon}(., t)=: \Delta_{2}, \\
& z_{0}=\Phi\left(y_{0}\right) \text { and } z_{1}=\Phi\left(y_{1}\right) \text { for } \Phi(y):=\left(p_{c}^{+}\right)^{-1}\left(p_{c}^{-}(y)\right) .
\end{aligned}
$$

They imply

$$
y_{2}=y_{1}+\Delta_{1}=\Phi^{-1}\left(z_{0}+\Delta_{2}\right)+\Delta_{1}=\Phi^{-1}\left(\Phi\left(y_{0}\right)+\Delta_{2}\right)+\Delta_{1} .
$$

Since $\Phi$ and its inverse $\Phi^{-1}$ have a bounded derivative on $\{y \geq \delta\}$ we conclude the local estimate

$$
\left|y_{2}-y_{0}\right| \leq C_{L}(\delta)\left(\left|\Delta_{1}\right|+\left|\Delta_{2}\right|\right) \leq C_{L} \int_{2 k \varepsilon+\varepsilon}^{2 k \varepsilon+3 \varepsilon}\left|\partial_{x} u^{\varepsilon}(., t)\right| \mathbf{1}_{\Gamma^{\varepsilon}} .
$$

Adding the inequalities (3.19) from $k=(a-\varepsilon) /(2 \varepsilon)$ to $k^{\prime}=(b-\varepsilon) /(2 \varepsilon)-1$, we find

$$
\begin{aligned}
\left|u^{\varepsilon}(b-0, t)-u^{\varepsilon}(a-0, t)\right| & \leq C_{L} \int_{a}^{b}\left|\partial_{x} u^{\varepsilon}(., t)\right| \mathbf{1}_{\Gamma^{\varepsilon}} \\
& \leq C_{L}|b-a|^{1 / 2}\left(\int_{a}^{L}\left|\partial_{x} u^{\varepsilon}(., t)\right|^{2} \mathbf{1}_{\Gamma^{\varepsilon}}\right)^{1 / 2} .
\end{aligned}
$$

This is estimate (3.17).

Implication (3.18) on jumps. Let $C_{0}$ be fixed and let $t$ be a time instance with $\left\|\partial_{x} u^{\varepsilon}(., t) \mathbf{1}_{\left\{u^{\varepsilon} \geq \delta / 2\right\}} \mathbf{1}_{\Gamma^{\varepsilon}}\right\|_{L^{2}}^{2} \leq C_{0}$. As shown in (3.19), we have the estimate

$$
\begin{aligned}
\left|u^{\varepsilon}(2 k \varepsilon+\varepsilon-0)-u^{\varepsilon}(2 k \varepsilon-\varepsilon-0)\right| & \leq c\left(\int_{2 k \varepsilon-\varepsilon}^{2 k \varepsilon+\varepsilon}\left|\partial_{x} u^{\varepsilon}(., t)\right|^{2} \mathbf{1}_{\Gamma^{\varepsilon}}\right)^{1 / 2} \sqrt{\varepsilon} \\
& \leq c C_{0} \sqrt{\varepsilon},
\end{aligned}
$$

Copyright (c) by SIAM. Unauthorized reproduction of this article is prohibited. 


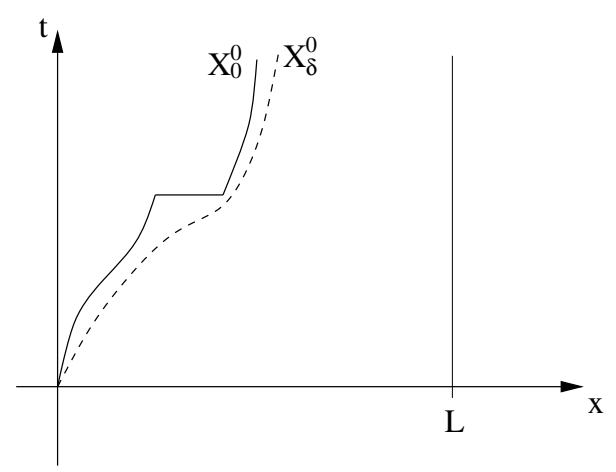

FIG. 6. Possible shapes of the free boundaries $X_{\delta}^{0}$ and $X_{0}^{0}$. We know that they are monotone and that $X_{\delta}^{0} \rightarrow X_{0}^{0}$ pointwise almost everywhere. With the above graphs we illustrate that $X_{0}^{0}$ may have jumps and that we cannot expect $X_{\delta}^{0}(t) \rightarrow X_{0}^{0}(t)$ for every $t>0$.

at least if we can assume (for the last inequality) that the saturation satisfies $u^{\varepsilon} \geq \delta / 2$ in every point of the interval $(2 k \varepsilon-\varepsilon, 2 k \varepsilon+\varepsilon)$. We choose $\varepsilon_{0}=\varepsilon_{0}\left(\delta, C_{0}\right)$ such that $c C_{0} \sqrt{\varepsilon_{0}} \leq \delta / 4$. The arguments above can be repeated for every point $x$ in the interval $(2 k \varepsilon, 2 k \varepsilon+\varepsilon)$. Continuity of $u^{\varepsilon}$ inside the interval allows us to conclude that $u^{\varepsilon}(2 k \varepsilon+0) \geq 3 \delta / 4$. We repeat the argument on the interval $(2 k \varepsilon-\varepsilon, 2 k \varepsilon)$ and find the result.

4. The free boundary problem. We study, for $\delta>0$ and $\varepsilon>0$, the free boundary separating the region of uniformly positive saturation from the rest:

$$
\begin{aligned}
& X_{\delta}^{\varepsilon}(t):=\inf \left\{x \in(0, L) \cap(2 \varepsilon \mathbb{Z}+\varepsilon): u^{\varepsilon}(x-0, t) \geq \delta\right\}, \\
& X_{0}^{\varepsilon}(t):=\inf _{\delta>0} X_{\delta}^{\varepsilon}(t) .
\end{aligned}
$$

We set $X_{\delta}^{\varepsilon}(t)=L$ if the infimum is taken over the empty set.

Lemma 6. There are sequences $\varepsilon_{k} \searrow 0$ and $\delta_{m} \searrow 0$ such that, for every $\varepsilon=\varepsilon_{k}$ and every $\delta=\delta_{m}$, the following hold:

1. The maps $t \mapsto X_{\delta}^{\varepsilon}(t)$ and $t \mapsto X_{0}^{\varepsilon}(t)$ are monotonically nondecreasing.

2. The following limits hold pointwise for almost every $t$, and the limits are monotone functions:

$$
\begin{array}{ll}
X_{\delta}^{0}(t)=\lim _{k \rightarrow \infty} X_{\delta}^{\varepsilon_{k}}(t), & X_{0}^{0}(t)=\lim _{m \rightarrow \infty} X_{\delta_{m}}^{0}(t), \\
X_{0}^{\varepsilon}(t)=\lim _{m \rightarrow \infty} X_{\delta_{m}}^{\varepsilon}(t), & \tilde{X}_{0}^{0}(t)=\lim _{k \rightarrow \infty} X_{0}^{\varepsilon_{k}}(t) .
\end{array}
$$

We can select an upper semicontinuous representative $t \mapsto X(t)$ of the $L^{1}$ function $t \mapsto X_{0}^{0}(t)$.

3. There holds $X_{\delta}^{0} \leq X_{\delta^{\prime}}^{0}$ for all $\delta \leq \delta^{\prime}$ and $\tilde{X}_{0}^{0} \leq X_{0}^{0}$.

Proof. Figure 6 indicates possible shapes of $X_{0}^{0}$ and $X_{\delta}^{0}$ and recalls the fact that these functions need not be continuous. Lemma 3 provides that the function $t \mapsto u^{\varepsilon}(x, t)$ is monotonically nonincreasing. This implies the monotonicity of the free boundaries stated in statement 1 . The monotonicity of the family of functions $t \mapsto X_{\delta}^{\varepsilon}$ implies the uniform boundedness in $B V([0, T], \mathbb{R})$, and hence we can extract subsequences that converge strongly in $L^{1}$ and pointwise almost everywhere. Limits of monotone functions are again monotone. Since $B V$-functions have only countably many jumps, we find an upper semicontinuous representative. 
The monotonicity in $\delta$ is an immediate consequence of the definition of $X_{\delta}^{\varepsilon}$. It justifies the infimum of (4.2) and implies $X_{0}^{\varepsilon} \leq X_{\delta}^{\varepsilon}$, which carries over in the limit $k \rightarrow \infty$ as $\tilde{X}_{0}^{0} \leq X_{\delta}^{0}$. The limit $\delta_{m} \rightarrow 0$ yields statement 3 .

With the help of the limiting free boundaries we can transform the results of Proposition 1 into the following statement.

Corollary 1 (limit equations in region $G$ ). Let $t \mapsto X(t)$ be as in Lemma 6 and $G$ the open domain:

$$
G:=\{(x, t) \in(0, L) \times(0, T): x>X(t)\} .
$$

Let $\left(u^{0}, F^{0}\right)$ be limits of entropy solutions $\left(u^{\varepsilon}, F^{\varepsilon}\right)$ as in Theorem 1 and $G^{\prime} \Subset G$ a subset of $G$. Then there holds $\partial_{x} u^{0} \in L^{2}\left(G^{\prime}\right)$ and $F^{0}$ satisfies on $G^{\prime}$ the relation $F^{0}=\mathcal{F}\left(u^{0}, \partial_{x} u^{0}\right)$.

Proof. The function $X$ is monotone and the closure of $G^{\prime}$ is a compact subset of $G$; hence $G^{\prime}$ can be covered by a finite collection of sets $G_{0}=\left(x_{0}, L\right) \times\left(0, t_{0}\right)$ with $x_{0}>X\left(t_{0}\right)$. It suffices to verify the statements on one such subset $G_{0}$. Our aim is to find $\varepsilon_{0}>0$ and $\delta>0$ such that $u^{\varepsilon} \geq \delta$ on $G_{0}$ for all $\varepsilon \leq \varepsilon_{0}$. Once this is done, the application of Proposition 1 yields the result.

We start by choosing $\eta>0$ such that $x_{0}-\eta>X\left(t_{0}+\eta\right)$, which is possible, since $X$ is upper semicontinuous, and hence $\lim \sup _{\eta \backslash 0} X\left(t_{0}+\eta\right) \leq X\left(t_{0}\right)<x_{0}$. We now choose $\delta>0$ such that

$$
x_{0}-\frac{\eta}{2}>X_{\delta}^{0}\left(t_{0}+\frac{\eta}{2}\right) .
$$

In order to verify that for a small $\delta>0$ relation (4.3) is satisfied, we exploit that by monotonicity $X(t)<x_{0}-\eta$ for all $t \in\left(t_{0}+\eta / 2, t_{0}+\eta\right)$. The strong $L^{2}$-convergence $X_{\delta}^{0} \rightarrow X$ and Egoroff's theorem imply that, for $\delta>0$ small, $\left|X_{\delta}^{0}-X\right|<\eta / 2$ except for a set of $t$ 's with measure less than $\eta / 2$. For such $\delta$ there necessarily exists $s \in$ $\left(t_{0}+\eta / 2, t_{0}+\eta\right)$ with $X_{\delta}^{0}(s)<x_{0}-\eta / 2$. By monotonicity of $X_{\delta}^{0}$, relation (4.3) holds.

We finally want to choose, in a similar way, a number $\varepsilon_{0}>0$ with $x_{0}>X_{\delta}^{\varepsilon}\left(t_{0}\right)$. We have $X_{\delta}^{0}(t)<x_{0}-\eta / 2$ for all $t \in\left(t_{0}, t_{0}+\eta / 2\right)$. By Egoroff's theorem we find $\varepsilon_{0}>0$ such that, for all $\varepsilon \leq \varepsilon_{0}$, we find some $s \in\left(t_{0}, t_{0}+\eta / 2\right)$ such that $X_{\delta}^{\varepsilon}(s)<x_{0}$. The monotonicity of $X_{\delta}^{\varepsilon}(s)$ implies $x_{0}>X_{\delta}^{\varepsilon}\left(t_{0}\right)$ and thus $G_{0} \subset\left(X_{\delta}^{\varepsilon}\left(t_{0}\right), L\right) \times\left(0, t_{0}\right)$. The definition of $X_{\delta}^{\varepsilon}$ implies the desired lower bound for the sequence $u^{\varepsilon}$ on the left boundary of the domain $\left(X_{\delta}^{\varepsilon}\left(t_{0}\right), L\right) \times\left(0, t_{0}\right)$. Lemma 2 yields the lower bound on the whole domain.

Proposition 2 (limit equations in region $B$ ). In the domain

$$
B:=\{(x, t) \in(0, L) \times(0, T): x \leq X(t)\},
$$

there holds that $u^{0} \equiv \frac{u^{*}}{2}$ and $F^{0} \equiv 0$ almost everywhere. The function $u^{0}$ has no jump across $\partial B \cap \Omega_{T}$ in the following sense: Let $T_{0} \in(0, T]$ be a time instance with $X\left(T_{0}\right)<L$, and let $A_{r}, r>0$, be a family of averages of $u^{0}$,

$$
A_{r}:=\frac{1}{T_{0} \cdot r} \int_{0}^{T_{0}} \int_{0}^{r} u^{0}(X(t)+s, t) d s d t .
$$

Then $A_{r}$ satisfies

$$
A_{r} \rightarrow \frac{u^{*}}{2} \text { for } r \rightarrow 0 .
$$

Copyright $@$ by SIAM. Unauthorized reproduction of this article is prohibited. 
Proof. We select monotone sequences $\delta_{j} \rightarrow 0$ and $\varepsilon_{m} \rightarrow 0$ with the convergences of the free boundaries as in Lemma 6 and with $u^{\varepsilon_{m}} \rightarrow u^{0}$ weakly in $L^{2}\left(\Omega_{T}\right)$. Almost all time instances $t \in(0, T)$ are points of continuity of the function $X($.$) and of all$ functions $X_{\delta_{j}}^{0}(),. j \in \mathbb{N}$. Furthermore, in almost every point $t \in(0, T)$ the convergences of Lemma 6 hold. In the following we consider only time instances $t$ with all these properties.

Step 1 . On $B$ it holds that $u^{0}=u^{*} / 2$. We note that $u^{0} \geq u^{*} / 2$ follows immediately from the lower bound in Lemma 4 .

For the upper bound let $(x, t) \in B$ be with $t$ as above and with $x<X(t)$. Moreover, let $\rho>0$ be arbitrary. We choose $\tau>0$ and an index $j \in \mathbb{N}$ such that, for $\delta=\delta_{j}$, implication (2.5) of Lemma 4 holds. Since $X$ has no jump in $t$ we find $t^{\prime}<t$ with $x<X\left(t^{\prime}\right)$. By monotonicity in $\delta$, we have $x<X_{\delta}^{0}\left(t^{\prime}\right)$. We choose $r>0$ smaller than $\frac{1}{2}\left(X_{\delta}^{0}\left(t^{\prime}\right)-x\right)$; for later use we also demand $r<\frac{1}{2}\left(t-t^{\prime}\right)$. We find $m_{0}>0$ such that, for all $m \geq m_{0}$, additionally $r<X_{\delta}^{\varepsilon_{m}}\left(t^{\prime}\right)-x$. We may choose $m_{0}$ large enough to satisfy additionally $\tau \varepsilon_{m_{0}}^{2}<t-t^{\prime}-r$ and $\varepsilon_{m_{0}}<\varepsilon_{0}$ of Lemma 4 . The upper bound of Lemma 4 provides, for $\varepsilon=\varepsilon_{m}$ with $m \geq m_{0}$,

$$
u^{\varepsilon}\left(2 k \varepsilon+\varepsilon-0, t^{\prime}\right) \leq \delta \Rightarrow \int_{2 k \varepsilon-\varepsilon}^{2 k \varepsilon+\varepsilon} u^{\varepsilon}\left(., t^{\prime}+\tau \varepsilon^{2}\right) \leq \varepsilon\left(u^{*}+\rho\right) .
$$

By construction, the assertion is satisfied for all $k \in \mathbb{Z}$ with $2 k \varepsilon+\varepsilon \leq x+r$. The monotone decay of $u^{\varepsilon}$ in $t$ implies

$$
\frac{1}{\left|B_{r}((x, t))\right|} \int_{B_{r}((x, t))} u^{\varepsilon_{m}} \leq \frac{1}{2}\left(u^{*}+\rho\right)
$$

This carries over to the weak limit $u^{0}$. Since $\rho$ is arbitrary, we have the upper bound in $B$ by the Lebesgue differentiation theorem.

Step 2. Boundary condition. We assume that a small number $\rho>0$ is given; our aim is to choose $r>0$ small to have $A_{r} \leq u^{*} / 2+c \rho$ for some universal constant $c$. We recall that $A_{r}$ is defined with an integration over the thin region

$$
U_{r}:=\left\{(x, t) \in \Omega_{T_{0}}: X(t)<x<X(t)+r\right\} .
$$

We use the numbers $\delta_{0}>0$ and $\tau>0$ that appear in the upper bound for averages in Lemma 4 and choose $\delta=\delta_{j}<\delta_{0} / 2$. We consider the $\varepsilon$-dependent set

$$
E_{0}:=\left\{(x, t) \in \Omega_{T_{0}}: x \in(0, L), \int_{0}^{L}\left|\partial_{x} u^{\varepsilon}(., t)\right|^{2} \mathbf{1}_{\left\{u^{\varepsilon} \geq \delta / 2\right\}}>C_{0}^{2}\right\},
$$

where we denote by $\partial_{x} u^{\varepsilon}$ the regular part of the derivative. Choosing $C_{0}$ large enough we achieve $\left|E_{0} \cap U_{r}\right| \leq \rho r T_{0}$ for all $\varepsilon$. This is possible since by estimate (2.1) the time integral over the above spatial integral is bounded. We now choose $r>0$ small enough to satisfy, with $C_{L}=C_{L}(\delta / 2)$ of Lemma $5, C_{L} C_{0}(4 r)^{1 / 2} \leq \delta$.

In order to show the upper bound for $A_{r}$ we may still choose $\varepsilon>0$ small. We define further $\varepsilon$-dependent exceptional sets as

$$
\begin{aligned}
& E_{1}:=\left\{(x, t) \in \Omega_{T_{0}}: x \in(0, L),\left|X_{\delta}^{\varepsilon}(t)-X_{\delta}^{0}(t)\right| \geq r\right\}, \\
& E_{1}^{\prime}:=\left\{(x, t) \in \Omega_{T_{0}}: x \in(0, L), t \geq \tau \varepsilon^{2},\left|X_{\delta}^{\varepsilon}\left(t-\tau \varepsilon^{2}\right)-X_{\delta}^{0}\left(t-\tau \varepsilon^{2}\right)\right| \geq r\right\}, \\
& E_{2}:=\left\{(x, t) \in \Omega_{T_{0}}: X_{\delta}^{\varepsilon}\left(t-\tau \varepsilon^{2}\right) \leq x \leq X_{\delta}^{\varepsilon}(t)\right\}, \\
& E_{3}:=\left\{(x, t) \in \Omega_{T_{0}}:\left|X(t)-X\left(t-\tau \varepsilon^{2}\right)\right| \geq r\right\} .
\end{aligned}
$$


For the first set we achieve $\left|E_{1}\right| \leq \rho r T_{0}$ for all small $\varepsilon$ by the $L^{1}$-convergence $X_{\delta}^{\varepsilon} \rightarrow X_{\delta}^{0}$. The set $E_{1}^{\prime}$ is obtained from $E_{1}$ by a shift, and hence this set also satisfies $\left|E_{1}^{\prime}\right| \leq \rho r T_{0}$. The set $E_{2}$ is contained in a $\tau \varepsilon^{2}$-neighborhood of the free boundary

$$
\Sigma_{\delta}^{\varepsilon}:=\left\{(x, t) \in \Omega_{T_{0}}: \lim _{s \nearrow t} X_{\delta}^{\varepsilon}(s) \leq x \leq \lim _{s \searrow t} X_{\delta}^{\varepsilon}(s)\right\} .
$$

The set $\Sigma_{\delta}^{\varepsilon}$ is a curve of finite length, and hence we achieve $\left|E_{2}\right| \leq \rho r T_{0}$ for $\varepsilon>0$ small. Finally, $\left|E_{3}\right| \leq \rho r T_{0}$ for $\varepsilon>0$ small, since $X$ is a $B V$-function. We may additionally impose on $\varepsilon$ that $\varepsilon<r$ and $\varepsilon<\varepsilon_{0}\left(\delta, C_{0}\right)$; the latter allows us to use the implication of Lemma 5 outside the set $E_{0}$,

$$
u^{\varepsilon}(2 k \varepsilon+\varepsilon-0, t) \geq \delta \quad \Rightarrow \quad u^{\varepsilon}(2 k \varepsilon-\varepsilon-0, t) \geq \delta / 2 .
$$

We note that the set

$$
E_{0}^{\prime}:=\left\{(x, t) \in(0, L) \times\left(\tau \varepsilon^{2}, T_{0}\right): \int_{0}^{L}\left|\partial_{x} u^{\varepsilon}\left(., t-\tau \varepsilon^{2}\right)\right|^{2} \mathbf{1}_{\left\{u^{\varepsilon}\left(., t-\tau \varepsilon^{2}\right) \geq \delta / 2\right\}}>C_{0}^{2}\right\}
$$

also satisfies $\left|E_{0}^{\prime} \cap U_{r}\right| \leq \rho r T_{0}$, since it is obtained by a shift of the set $E_{0}$.

After these preparations, let us now consider an arbitrary point $(x, t)=(2 k \varepsilon+$ $\varepsilon, t) \in U_{r} \backslash\left(E_{0} \cup E_{0}^{\prime} \cup E_{1} \cup E_{1}^{\prime} \cup E_{2} \cup E_{3}\right)$. We distinguish two cases.

Case (i). $u^{\varepsilon}(x, t)$ is small, $x=2 k \varepsilon+\varepsilon<X_{\delta}^{\varepsilon}(t)$. Since $(x, t)$ is not contained in $E_{2}$, we also have $x<X_{\delta}^{\varepsilon}\left(t-\tau \varepsilon^{2}\right)$. Lemma 4 can be applied with the point $\left(2 k \varepsilon+\varepsilon, t-\varepsilon^{2} \tau\right)$ and yields

$$
\int_{2 k \varepsilon-\varepsilon}^{2 k \varepsilon+\varepsilon} u^{\varepsilon}(., t) \leq \varepsilon\left(u^{*}+\rho\right)
$$

Case (ii). $u^{\varepsilon}(x, t)$ is large, $x=2 k \varepsilon+\varepsilon \geq X_{\delta}^{\varepsilon}(t)$. We will derive the smallness of $u^{\varepsilon}(x, t)$ with the help of the Hölder-type estimate of Lemma 5 and conclude again with Lemma 4.

We start by setting $t^{\prime}:=t-\tau \varepsilon^{2}$ and denote by $k^{\prime}$ the integer with $2 k^{\prime} \varepsilon+\varepsilon=$ $x^{\prime}:=X_{\delta}^{\varepsilon}\left(t^{\prime}\right)$. The definition of $X_{\delta}^{\varepsilon}$ implies $u^{\varepsilon}\left(x^{\prime}-0, t^{\prime}\right) \geq \delta,\left(x^{\prime}, t^{\prime}\right) \notin E_{0}^{\prime}$ allows us to use (4.6) at the time instance $t^{\prime}$, and we conclude that $\delta / 2 \leq u^{\varepsilon}\left(2 k^{\prime} \varepsilon-\varepsilon-0, t^{\prime}\right)<\delta$. The lower bound allows us to apply the first part of Lemma 5 with $a=2 k^{\prime} \varepsilon-\varepsilon$ and $b=x=2 k \varepsilon+\varepsilon$. We find

$$
u^{\varepsilon}\left(x-0, t^{\prime}\right) \leq u^{\varepsilon}\left(2 k^{\prime} \varepsilon-\varepsilon-0, t^{\prime}\right)+C_{L}(\delta / 2) C_{0}(4 r)^{1 / 2} \leq \delta+\delta=2 \delta .
$$

We used here $(x, t) \notin E_{3}$ and $(x, t) \notin E_{1}^{\prime}$ such that

$$
\begin{aligned}
x-x^{\prime} & =(x-X(t))+\left(X(t)-X\left(t^{\prime}\right)\right)+\left(X\left(t^{\prime}\right)-X_{\delta}^{0}\left(t^{\prime}\right)\right)+\left(X_{\delta}^{0}\left(t^{\prime}\right)-X_{\delta}^{\varepsilon}\left(t^{\prime}\right)\right) \\
& \leq r+r+0+r=3 r .
\end{aligned}
$$

Another application of the upper bound of Lemma 4 yields also in this case

$$
\int_{2 k \varepsilon-\varepsilon}^{2 k \varepsilon+\varepsilon} u^{\varepsilon}(., t) \leq \varepsilon\left(u^{*}+\rho\right) .
$$

In both cases we find the same estimate for averages of $u^{\varepsilon}$. Summation over $k$ and an integration over $t \in\left(0, T_{0}\right)$ yield

$$
\frac{1}{T_{0} \cdot r} \int_{U_{r}} u^{\varepsilon} \leq \frac{u^{*}}{2}+c \rho+O(\varepsilon) \frac{1}{T_{0} \cdot r} .
$$

Copyright $@$ by SIAM. Unauthorized reproduction of this article is prohibited. 
The factor $c$ covers the error induced by the exceptional sets, and the error term $O(\varepsilon)$ is induced by the integration over a boundary strip of width $2 \varepsilon$ that is necessary to cover $U_{r}$ with intervals of the form $(2 k \varepsilon, 2 k \varepsilon+2 \varepsilon)$. We take the limit $\varepsilon \rightarrow 0$. Since $\rho>0$ is arbitrary, we find (4.4).

Step 3. On $B$ it holds that $F^{0}=0$. We have shown in Step 1 that $u^{0}$ is constant in $B$, and hence the conservation law implies $\partial_{x} F^{0}=0$ and we have $F^{0}(x, t)=F^{0}(t)$ for almost all $(x, t) \in B$. Our aim is to conclude $F^{0}(t)=0$ for almost all $t$.

Inequality $F^{0} \leq 0$. For the approximate solutions $u^{\varepsilon}$, the boundary condition $u^{\varepsilon}(0, t)=0$, together with $f(0)=0, g \geq 0$, and $u^{\varepsilon} \geq 0$, implies $F^{\varepsilon}(0, t)=$ trace $\left\{f\left(u^{\varepsilon}\right)-g\left(u^{\varepsilon}\right) \partial_{x} u^{\varepsilon}\right\} \leq 0$. This can be written in a weak form as

$$
\int_{0}^{T} \int_{0}^{L}\left\{u^{\varepsilon} \cdot \partial_{t} \varphi+F^{\varepsilon} \cdot \partial_{x} \varphi\right\} \geq 0 \quad \forall \varphi \in C_{0}^{\infty}((0, T) \times[0, L)), \varphi \geq 0 .
$$

We can take the limit $\varepsilon \rightarrow 0$ in these integrals and conclude that $F^{0} \leq 0$ on $B$ from $\partial_{t} u^{0}=0$.

Inequality $F^{0} \geq 0$. This inequality is not a consequence of the boundary conditions but must be concluded with the help of the positivity of the global convection term $f(u)$. We consider an arbitrary rectangle $U \Subset B$ and a number $q>0$ and show for some $m_{0}$ that

$$
F^{\varepsilon_{m}} \geq-q \text { on } U \text { for all } m \geq m_{0} .
$$

Once this is shown, we have $F^{0} \geq 0$ almost everywhere on $B$.

We fix the rectangle $U \Subset B$ and the number $q>0$. We choose $\delta>0$ small compared to $q \cdot \inf \{x:(x, t) \in U\}>0$ and refer the reader to the end of the proof for the precise choice. We now select $m_{0}$ such that, for all $m \geq m_{0}$, (i) $x \leq X_{\delta / 2}^{\varepsilon}(t)$ for all $(x, t) \in U$, (ii) $u^{\varepsilon}(x, t) \leq \delta$ for all $(x, t) \in U$ with $x \in \Gamma_{-}^{\varepsilon}$, (iii) $u^{*} / 2 \leq u^{\varepsilon}(x, t) \leq$ $\left(u^{*}+1\right) / 2$ for all $(x, t) \in U$ with $x \in \Gamma_{-}^{\varepsilon}$. The existence of such an $m_{0}$ follows from the $B V$-convergence $X_{\delta / 2}^{\varepsilon} \rightarrow X_{\delta / 2}^{0}$, the argument of (3.18), and the lower bound for averages. We now assume that, for some $(x, t) \in U$ and some $m \geq m_{0}$,

$$
F^{\varepsilon_{m}}(x, t)=f^{\varepsilon_{m}}\left(x, u^{\varepsilon_{m}}(x, t)\right)-g^{\varepsilon_{m}}\left(x, u^{\varepsilon_{m}}(x, t)\right) \partial_{x} u^{\varepsilon_{m}}(x, t)<-q
$$

and derive a contradiction.

Since $\partial_{x} F^{\varepsilon}=-\partial_{t} u^{\varepsilon}$ is nonnegative, $F^{\varepsilon}$ is monotonically increasing and we have

$$
-g^{\varepsilon}\left(x, u^{\varepsilon_{m}}\left(x^{\prime}, t\right)\right) \partial_{x} u^{\varepsilon}\left(x^{\prime}, t\right)=F^{\varepsilon}\left(x^{\prime}, t\right)-f^{\varepsilon}\left(x, u^{\varepsilon_{m}}\left(x^{\prime}, t\right)\right) \leq F^{\varepsilon}\left(x^{\prime}, t\right) \leq-q
$$

for all $x^{\prime} \in(0, x)$. This implies that $u^{\varepsilon}(., t)$ is increasing on $\Gamma_{-}^{\varepsilon} \cap(0, x)$, and on $\Gamma_{+}^{\varepsilon} \cap(0, x)$ it is strictly increasing with a lower bound

$$
\partial_{x} u^{\varepsilon} \geq \frac{q}{g^{*}} \text { with } g^{*}:=\sup _{\xi \in\left(u^{*} / 2,\left(1+u^{*}\right) / 2\right)} g(\xi)>0 .
$$

The monotonicity of $p_{c}{ }^{ \pm}$implies that $u^{\varepsilon}$ is increasing on $(0, x) \cap 2 \varepsilon \mathbb{Z}$ with an average slope of at least $q / 2 g^{*}$. The boundary condition $u^{\varepsilon}(0, t)=0$ leads to

$$
u^{\varepsilon}(x, t) \geq \frac{q}{2 g^{*}} x>\delta
$$

if $\delta$ was chosen with $\delta<q \inf \{x:(x, t) \in U\} / 2 g^{*}$. This is in contradiction with $u^{\varepsilon}(x, t) \leq \delta$ of (ii). The proof of $F^{0}=0$ on $B$ is complete. 
Proof of Theorem 1. A priori estimates for $u^{\varepsilon}$ and $F^{\varepsilon}$ are shown in Lemma 1 of section 2, and we may therefore select weakly convergent subsequences. The weak convergences allow us to take the distributional limit in the conservation law and we find (1.12).

In Lemma 6 we constructed a monotone function $X:[0, T] \rightarrow \mathbb{R}$ such that $G=\{(x, t) \mid x>X(t)\} \subset \Omega_{T}$ is an open set. In Proposition 2 we derived $u^{0} \equiv u^{*} / 2$ and $F^{0}=0$ almost everywhere on $B:=\Omega_{T} \backslash G$; since $\mathcal{F}$ satisfies $\mathcal{F}\left(u^{*} / 2, \zeta\right)=0$ for all $\zeta \in \mathbb{R}$, (1.13) holds pointwise almost everywhere on $B$. Corollary 1 provides $\partial_{x} u^{0} \in L_{l o c}^{2}(G)$ and (1.13) on $G$.

We already know that $\partial_{x} u^{0}$ is an $L_{l o c}^{2}$-function on $\Omega_{T} \backslash \Sigma$ for $\Sigma=\partial G \cap \partial B$. Lemma 3 implies that $\partial_{x} u^{0}$ is nonnegative, and the boundedness of $u^{0}$ implies that the derivative $\partial_{x} u^{0}$ is a nonnegative measure on $\Omega_{T}$. Each slice $u^{0}(., t)$ is a $B V$ function, and hence the singular part of the measure $\partial_{x} u^{0}$ is concentrated on $\Sigma$ and regular with respect to the one-dimensional Hausdorff measure. Proposition 2 shows that this singular part of the measure $\partial_{x} u^{0}$ vanishes. We thus verified the statement $\partial_{x} u^{0} \in L^{1}\left(\Omega_{T}\right)$.

\section{REFERENCES}

[1] G. Allaire, Homogenization and two-scale convergence, SIAM J. Math. Anal., 23 (1992), pp. $1482-1518$.

[2] H. W. Alt and S. Luckhaus, Quasilinear elliptic-parabolic differential equations, Math. Z., 183 (1983), pp. 311-341.

[3] L. Ambrosio, N. Fusco, And D. Pallara, Functions of bounded variation and free discontinuity problems, Oxford Math. Monogr., Clarendon Press, Oxford University Press, New York, 2000.

[4] A. Beliaev, Homogenization of two-phase flows in porous media with hysteresis in the capillary relation, European J. Appl. Math., 14 (2003), pp. 61-84.

[5] M. Bertsch, R. Dal Passo, and C. J. van Duijn, Analysis of oil trapping in porous media flow, SIAM J. Math. Anal., 35 (2003), pp. 245-267.

[6] A. Bourgeat, S. M. Kozlov, and A. Mikelić, Effective equations of two-phase flow in random media, Calc. Var. Partial Differential Equations, 3 (1995), pp. 385-406.

[7] A. Bourgeat, S. Luckhaus, And A. Mikelić, Convergence of the homogenization process for a double-porosity model of immiscible two-phase flow, SIAM J. Math. Anal., 27 (1996), pp. 1520-1543.

[8] Z. CHEN, Degenerate two-phase incompressible flow. I. Existence, uniqueness and regularity of a weak solution, J. Differential Equations, 171 (2001), pp. 203-232.

[9] C. J. van Duijn, H. Eichel, R. Helmig, and I. S. Pop, Effective Equations for Two-Phase Flow in Porous Media: The Effect of Trapping at the Micro Scale, CASA Report 2005-35, Eindhoven, 2005.

[10] C. J. van DuiJn, A. Mikelić, And I. S. Pop, Effective equations for two-phase flow with trapping on the micro scale, SIAM J. Appl. Math., 62 (2002), pp. 1531-1568.

[11] B. H. GILDInG, The occurrence of interfaces in nonlinear diffusion-advection processes, Arch. Ration. Mech. Anal., 100 (1988), pp. 243-263.

[12] D. Kröner And S. Luckhaus, Flow of oil and water in a porous medium, J. Differential Equations, 55 (1984), pp. 276-288.

[13] B. H. Kueper And E. O. Frind, Two-phase flow in heterogeneous porous media: 1. Model development, Water Resources Research, 27 (1991), pp. 1049-1057.

[14] P. VAn Lingen, Quantification and Reduction of Capillary Entrapment in Cross-Laminated Oil Reservoirs, Ph.D. thesis, Delft University of Technology, Delft, 1998.

[15] F. Отто, $L^{1}$-contraction and uniqueness for quasilinear elliptic-parabolic equations, J. Differential Equations, 131 (1996), pp. 20-38.

[16] B. SchweIzer, Regularization of outflow problems in unsaturated porous media with dry regions, J. Differential Equations, 237 (2007), pp. 278-306.

[17] B. Schweizer, Averaging of flows with capillary hysteresis in stochastic porous media, European J. Appl. Math., 18 (2007), pp. 389-415. 